\title{
Tapentadol Prolonged Release Versus Strong Opioids for Severe, Chronic Low Back Pain: Results of an Open-Label, Phase 3b Study
}

Rafael Gálvez · Michael Schäfer · Guy Hans · Dietmar Falke · Ilona Steigerwald

To view enhanced content go to www.advancesintherapy.com

Received: December 22, 2012 / Published online: March 7, 2013

(C) The Author(s) 2013. This article is published with open access at Springerlink.com

\section{ABSTRACT}

Introduction: This open-label, phase $3 \mathrm{~b}$ study evaluated the effectiveness and tolerability of oral tapentadol prolonged release (PR; 50-250 mg twice daily [b.i.d.]) for managing severe, chronic low back pain in patients responding to World

ClinicalTrials.gov \#NCT00986258.

R. Gálvez

Unit of Pain and Palliative Care, Hospital Universitario Virgen de las Nieves, Granada, Spain

M. Schäfer

Department of Anaesthesiology and Intensive Care Medicine, Charité University, Campus Virchow

Klinikum, Berlin, Germany

G. Hans

Multidisciplinary Pain Center (PCT), University of

Antwerp, Wilrijk, Belgium

D. Falke $\cdot$ I. Steigerwald $(\bowtie)$

Medical Affairs Europe \& Australia, Grünenthal GmbH, Zieglerstrasse 6, 52078 Aachen, Germany e-mail: ilona.steigerwald@grunenthal.com

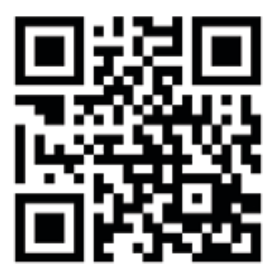

Enhanced content for Advances in Therapy articles is available on the journal web site: www.advancesintherapy.com
Health Organization (WHO) step III opioids but tolerating treatment poorly. Equianalgesic ratios for tapentadol to prior strong opioids were calculated.

Methods: Patients rotated directly from prior WHO step III opioids to tapentadol. Patients received tapentadol PR (50-250 mg b.i.d.) during 5-week titration and 7-week maintenance periods. Tapentadol immediate release (IR) $50 \mathrm{mg}$ ( $\leq$ twice/day, $\geq 4 \mathrm{~h}$ apart) was allowed (total daily dose of tapentadol PR and IR $\leq 500 \mathrm{mg}$ /day). The primary endpoint was responder rate 1 at week 6 (percentage of patients with the same or less pain intensity [11-point numerical rating scale (NRS; 3-day average)] vs week -1).

Results: Responder rate 1 at week 6 (last observation carried forward [LOCF]) was $80.9 \%$ (76/94; $P<0.0001$ vs. the null responder hypothesis rate $[<60 \%]$ ), resulting in a positive trial despite premature termination (136 recruited of 180 planned). Significant improvements from baseline in pain intensity and neuropathic pain symptoms were observed at weeks 6 and 12 with tapentadol PR $(P<0.05)$. Equianalgesic ratios were calculated for PR formulations alone and for PR and IR formulations combined for tapentadol to oxycodone, buprenorphine, fentanyl, morphine, 
and hydromorphone. The prevalences of adverse events reported as the reason for switching to tapentadol (most commonly constipation and nausea) decreased over time.

Conclusions: Tapentadol PR (50-250 mg b.i.d.) provided at least comparable pain relief and improved tolerability versus prior strong opioids in patients with severe, chronic low back pain responding to WHO step III therapy. Conversion from strong opioids to tapentadol PR, with its two mechanisms of action, went smoothly considering overall effectiveness and tolerability outcomes. Equianalgesic ratios of tapentadol to oxycodone and other strong opioids were in line with other phase $3 / 3 \mathrm{~b}$ studies.

Keywords: Chronic pain; Equianalgesic; Equipotency; Low back pain; Neuropathic pain; Opioid; Severe pain; Tapentadol

\section{INTRODUCTION}

Opioid analgesics are recommended for managing severe, chronic low back pain $[1,2]$. Opioid treatment may be associated with side effects [3] that may be the first reason leading patients to discontinue opioid therapy $[4,5]$ or rotate to a different opioid. Gastrointestinal side effects, including nausea, constipation, and vomiting, are among the most commonly reported opioid-related side effects [4], and patients have reported that these are the most bothersome side effects associated with opioid treatment $[6,7]$. Patients often miss or decrease their dose of opioid analgesic or discontinue opioid treatment to avoid nausea, vomiting, and constipation $[7,8]$, and physicians often discontinue opioid therapy because of these side effects [9]. In a survey [8] of 322 patients taking daily oral opioids and laxatives, 33\% of patients had skipped, decreased, or stopped their opioid analgesic in order to obtain relief from opioid-induced constipation. In addition, opioid analgesics may not be effective for all patients with chronic pain, particularly patients with a neuropathic pain component to their low back pain (e.g., radiculopathy), which may not respond particularly well to opioid analgesics alone $[10,11]$. Chronic therapy with opioid analgesics may result in development of tolerance, such that patients require higher average doses to maintain effective analgesia but potentially leading to more side effects [12]. Such tolerance development is another major reason why patients require opioid rotation [12]. The vast majority of physicians ( $>90 \%)$ report using combination treatment rather than monotherapy for the management of severe, chronic pain [13]; however, a recent Cochrane review by Chaparro and colleagues [14] of randomized controlled trials evaluating combination therapy for neuropathic pain found that although combination therapy offered similar or sometimes a modest gain in efficacy, it was often associated with higher incidences of side effects and discontinuations due to side effects.

The centrally acting analgesic tapentadol has two mechanisms of action, $\mu$-opioid receptor agonism and noradrenaline reuptake inhibition $[15,16]$. Tapentadol oral prolonged release (PR; 100-250 mg twice daily [b.i.d.]) has been shown to be effective and well tolerated for the management of moderate to severe, chronic pain in previous phase 3 studies [17-21], including randomized, doubleblind, placebo-controlled studies in patients with low back pain [18] and pain related to diabetic peripheral neuropathy [20]. A pooled analysis [19] of efficacy and tolerability results from phase 3 studies of tapentadol for moderate to severe, chronic osteoarthritis knee pain or low back pain showed that tapentadol PR (100-250 mg b.i.d.) provided comparable 
analgesic efficacy to oxycodone hydrochloride ( $\mathrm{HCl})$ controlled release (20-50 mg b.i.d.) and that tapentadol PR was associated with superior gastrointestinal tolerability to oxycodone controlled release (based on the incidences of nausea, vomiting, and constipation and fewer discontinuations, mainly related to gastrointestinal and central nervous system adverse events [AEs]). The effectiveness of tapentadol PR has also been demonstrated in a phase $3 b$ study of patients with severe low back pain with and without a neuropathic pain component that was not adequately managed with nonsteroidal anti-inflammatory drugs, paracetamol, or weak opioids (i.e., World Health Organization [WHO] step I or II analgesics) [22].

This multicenter, multinational, open-label phase $3 \mathrm{~b}$ study included patients with severe, chronic low back pain who had responded to WHO step III opioid therapy (i.e., a low pain level) but showed a lack of tolerability. The population of patients in this study had chronic low back pain that had responded to WHO step III therapy, which differs from a population of patients that suffered from pain following washout of prior analgesics or a population of predefined nonresponders suffering from lack of efficacy of their previous treatment. The main objective of this trial was to demonstrate comparable effectiveness for tapentadol PR to strong opioids in strong opioid responders who rotated to tapentadol directly from WHO step III opioids; thus, major improvements in measures of efficacy were not a main objective of the trial, but were captured and are described here. Equianalgesic ratios were determined for tapentadol versus WHO step III analgesics, including analgesics other than oxycodone, which has been the primary active comparator during clinical trials evaluating tapentadol.

\section{MATERIALS AND METHODS}

All procedures followed were in accordance with the ethical standards of the responsible committee on human experimentation (institutional and national) and with the Helsinki Declaration of 1975, as revised in 2000. Informed consent was obtained from all patients for inclusion in the study.

\section{Patient Population}

This study included men and nonpregnant, nonlactating women who were at least 18 years of age and had a diagnosis of chronic low back pain that had been present for a minimum of 3 months. If a radicular pain syndrome was present, it must have been present for a minimum of 3 months and stable for a minimum of 4 weeks prior to enrolling in the study. A WHO step III analgesic (in the opinion of the investigator) must have been required for the management of patients' low back pain. Patients must have been taking a WHO step III analgesic for a minimum of 3 months prior to screening and must have responded to that analgesic, as indicated by an average pain intensity score at screening of no more than 5 on an 11-point numerical rating scale-3 (NRS-3; average pain intensity [11-point NRS; $0=$ "no pain" to $10=$ "pain as bad as you can imagine"] over the 3 days prior to the pain intensity assessment). Eligible patients had to report opioid-related side effects as the reason for a change in their analgesic, and subject satisfaction with their previous treatment was not permitted to be better than "fair" on a 5-point verbal rating scale (VRS; $0=$ "poor," 1 = "fair," 2 = "good," 3 = "very good," 4 = "excellent").

Patients were excluded from the study if they had any concomitant painful conditions other than low back pain (e.g., anatomical deformities, fibromyalgia), had any clinically significant 
disease or laboratory finding, had active systemic or local infection, or required any painful procedures during the study (e.g., major surgery) that could confound or bias study assessments. Additional exclusion criteria included the following: a history or presence of alcohol or drug abuse; the presence of concomitant autoimmune inflammatory conditions; a history of or active hepatitis B or C within 3 months of screening; a history of HIV infection; a history of or laboratory values reflecting severe renal impairment; a history or presence of moderate or severe hepatic impairment; a history of seizure disorder or epilepsy; a history of severe traumatic brain injury within 15 years of screening or residual sequelae suggesting transient changes in consciousness; and a history of mild or moderate traumatic brain injury, stroke, transient ischemic attack, or brain neoplasm within 1 year of screening. Patients with a history of allergy or hypersensitivity to tapentadol or its excipients or contraindications to tapentadol (i.e., known or suspected paralytic ileus, acute or severe bronchial asthma or hypercapnia) were excluded from the study. Patients were also excluded from the study if they had taken monoamine oxidase inhibitors within 14 days prior to screening or nonstable doses of selective serotonin reuptake inhibitors (SSRIs) within 30 days prior to screening; SSRIs were permitted prior to and during the study if the dose had been stable for at least 30 days prior to screening and remained stable throughout the study.

\section{Study Design}

This was a multicenter, open-label, phase $3 \mathrm{~b}$ study that included a 1-week observation period (on the previous WHO step III analgesic regimen), a 5-week titration and stabilization period, and a 7-week maintenance period. Patients stopped taking all WHO step III analgesics and any potential concomitant WHO step II analgesics at the end of the observation period (week -1) before they received their first dose of study medication. A morphine equivalent dose (MED) of the previous WHO step III analgesic(s) was determined for each patient using the mean total daily dose (TDD) of all formulations of all WHO step III opioids taken during the 3 days prior to the baseline visit; that TDD was converted to a MED supported by the electronic case report form. At the start of the titration period, patients received a starting dose of tapentadol PR of $50 \mathrm{mg}, 100 \mathrm{mg}$, or $150 \mathrm{mg}$ b.i.d., depending on the MED of the previous WHO step III analgesic(s). A MED of up to $100 \mathrm{mg}$ per day corresponded to a starting tapentadol PR dose of $50 \mathrm{mg}$ b.i.d., a MED of 101-160 mg/day corresponded to a starting tapentadol PR dose of $100 \mathrm{mg}$ b.i.d., and a MED of more than $160 \mathrm{mg}$ per day corresponded to a starting tapentadol PR dose of $150 \mathrm{mg}$ b.i.d. In general, starting tapentadol PR doses were notably below the calculated MED.

At 3 days after the start of study medication (the time point corresponding to the interim visit), a first titration was permitted if needed. Doses of tapentadol PR were then titrated on a weekly basis within the dose range of tapentadol PR $50 \mathrm{mg}$ to $250 \mathrm{mg}$ b.i.d. to the dose that provided at least similar pain relief to the previous analgesic regimen, as indicated by a pain intensity score (11-point NRS-3) that was the same or lower than at baseline. Doses could then be titrated to achieve (or exceed) the following goals: at least a 1-point reduction in the pain intensity score (11-point NRS-3) from baseline and a subject satisfaction with treatment rating (5-point VRS) of at least "good."

Unless patients were participating in a tapering substudy (described below), doses of WHO step I analgesics and coanalgesics and medications used to control opioid-related side 
effects due to the previous analgesic regimen were kept stable throughout the titration and maintenance periods. During the titration period and the remainder of the study, tapentadol immediate release [IR] was permitted in doses of $50 \mathrm{mg}$ up to twice a day and at least $4 \mathrm{~h}$ apart for acute pain episodes due to index pain that had no clear cause, was related to increased activity or movement (incidental pain), or was related to end-of-dose failure (which indicated a need to adjust the dose of tapentadol PR) and for withdrawal symptoms, such as hyperalgesia, that might occur during the first days of the titration period after stopping treatment with the previous opioid and that were related to the previous opioid. Patients were not permitted to take combined TDD of tapentadol PR and tapentadol IR of more than $500 \mathrm{mg}$ per day. The dose of tapentadol PR determined during the titration period was continued during the maintenance period.

For patients who participated in the tapering substudy, concomitant WHO step I analgesics and coanalgesics (such as anticonvulsants and antidepressants) were tapered and stopped, whenever possible, between weeks 9 and 11, without compromising the pain relief achieved on tapentadol treatment. In that substudy, only one analgesic or coanalgesic was tapered, with coanalgesics preferentially tapered over WHO step I analgesics. Depending on the available doses and the starting dose, WHO step I analgesics and coanalgesics were tapered at weekly intervals in 1-3 steps or at 5-day intervals in four steps. If pain intensity (NRS-3) did not increase following a reduction in the dose of the WHO step I analgesic or coanalgesic, the dose was further reduced until the medication was stopped or until the pain intensity score increased. The dose of the WHO step I analgesic or coanalgesic was returned to the previous dose level if the pain intensity score increased.
Effectiveness, Function, Quality of Life, and Tolerability Evaluations

Safety and tolerability analyses were performed on all patients who took one or more doses of study medication (the safety population). The pain intensity, responder rate, and equianalgesic analyses described in this section were performed for both the main analysis and per-protocol populations; all other effectiveness, function, and quality-of-life analyses were performed only for the main analysis population. The main analysis population included all patients who took one or more doses of study medication and had one or more post-baseline pain intensity assessments, and the per-protocol population (which was a subset of the main analysis population) included all patients who had received treatment through week 6 and had no major protocol deviations.

Patients rated their pain intensity (11-point NRS-3) at screening, baseline, and all subsequent study visits. The primary endpoint for this study was responder rate 1 at week 6 in the perprotocol population, using the last observation carried forward (LOCF) to impute missing assessments; responder rate 1 was defined as the percentage of patients with the same or a lower pain intensity score compared with week -1 (when patients were on their previous WHO step III opioid). Responder rate 2 at week 6 (LOCF) in the per-protocol population was used as a secondary endpoint; responder rate 2 was defined as the percentage of patients with the same or a lower pain intensity score and an improvement in subject satisfaction with treatment of one category or more. Responder rates 1 and 2 were also analyzed in the main analysis population using observed-case analysis at weeks 6,8 , and 12 . The following assessments were also evaluated as secondary endpoints: the change in average pain intensity from week -1 
to weeks 6,8 , and 12 ; subject satisfaction with prior treatment (at screening and baseline) and study treatment (at the interim visit and all subsequent study visits; 5-point VRS); the patient global impression of change (PGIC) [23, 24], the clinician global impression of change (CGIC) [25], the EuroQol-5 Dimension (EQ-5D) health status questionnaire [26], the Short Form-36 (SF-36) health survey [27], and the Hospital Anxiety and Depression Scale (HADS) [28].

For the PGIC [23, 24] and CGIC [25], patients and investigators, respectively, rated their impression of the change in the patients' overall condition since baseline using a 7 -point numerical rating scale ( 1 = "very much improved" to 7 = "very much worse"). Both the PGIC and CGIC were completed at the interim visit and all subsequent study visits. For the EQ-5D health status questionnaire [26], patients rated five dimensions of health status (mobility, self-care, usual activities, pain/discomfort, and anxiety/depression) using one of three possible levels ("no problems," "some problems," or "extreme problems"). The SF-36 health status questionnaire [27] was used to assess eight different dimensions of health status (physical activities, role physical, bodily pain, general health, vitality, social functioning, role emotional, and mental health); each of the eight dimensions was scored on a scale from 0 ("poor health") to 100 ("good health"). For the HADS (used to assess anxiety and depression in medically compromised patients) [28], patients rated 14 questions that assessed different aspects of anxiety and depression on a 4-point scale (0-3), with higher scores indicating more severe anxiety or depression symptoms [29]. The EQ-5D health status questionnaire, the SF-36 health status questionnaire, and the HADS were completed at screening, baseline, week 1 , and at each study visit from week 5 through week 12 .
Total daily doses of tapentadol PR and tapentadol IR (both separately and overall) were recorded throughout the study, as were total daily doses of WHO step II and III opioids taken during week -1 and average doses of WHO step I analgesics and coanalgesics (average daily dose during the last $72 \mathrm{~h}$ [3 days] prior to the respective visit) taken throughout the study.

AEs were recorded throughout the study for the safety population and were classified as treatment-emergent AEs (TEAEs; AEs that newly occurred after the first intake of study medication or that increased in intensity, frequency, or quality after the first intake of study drug) and non-TEAEs (NTEAEs; AEs occurring or present [including ongoing medical history] before starting study medication [from screening to the first intake of study medication]). TEAEs were coded using the Medical Dictionary for Regulatory Activities (MedDRA), version 13.1. Any TEAE that resulted in death, was lifethreatening, required hospitalization or an increase in the duration of hospitalization, resulted in long-term or significant disability or incapacity, was a congenital anomaly or birth defect, or was considered medically important was considered a serious TEAE. The possible association of AEs with any WHO step III analgesics and coanalgesics was documented. AEs were also classified according to their relationship to the administration of study medication as not related or unlikely related if there was evidence of no causal relationship to the administration of study medication and at least possibly related if limited evidence of a causal relationship was shown. The prevalence of AEs that were reported as the underlying reason for switching to tapentadol was compared between week -1 (on prior WHO step III therapy) and week 12 (on tapentadol PR). Standard clinical laboratory evaluations and vital sign measurements were performed on the 
safety population at different time points during the study.

\section{Neuropathic Pain Component Evaluations}

The likelihood of a neuropathic pain component to low back pain was evaluated using the painDETECT questionnaire [30], which is a validated and highly sensitive screening tool that addresses the frequency and quality of neuropathic pain symptoms (seven items), pain patterns over time (one item), and radiating pain (one item). Scores for those nine items were summed to yield a total painDETECT score (possible score of 0-38) that was used to classify the likelihood of a neuropathic pain component to low back pain as "negative" (score of 0-12), "unclear" (score of 13-18), or "positive" (score of 19-38). The painDETECT questionnaire was completed at screening, at baseline, and at weeks 6,8 , and 12 . Patients with a painDETECT "negative" score at both the screening and baseline visit were included in the painDETECT negative subset. Patients with a painDETECT "positive" score at screening or baseline were included in the painDETECT positive subset. Patients with a painDETECT "unclear" score at screening and a "negative" score at baseline (or vice versa) were included in the painDETECT unclear subset.

For patients with a painDETECT "positive" score at baseline, a diagnosis of lumbar radiculopathy was made for those who had typical dermatomal pain radiating beyond the knee towards the foot (sciatica) and evoked by stretching within the distribution of the ischiadic/femoral nerve (positive Lasègue sign), along with one or more of the following signs of root dysfunction: sensory impairment, motor symptoms from compression of lumbar nerve root (L4, L5, S1), and/or absent or diminished quadriceps femoris or triceps surae reflexes, and/or signs of root dysfunction in quantitative sensory testing.

For patients in the painDETECT unclear or positive subset, the Neuropathic Pain Symptom Inventory (NPSI) [31] was used to further evaluate the neuropathic pain component of their low back pain. The NPSI referred to pain radiating towards or into the leg in this study and was completed at screening, baseline, and at each subsequent study visit. The NPSI [31] is a validated measure that consists of 10 questions that address different qualities of spontaneous, ongoing or paroxysmal pain, evoked pain, and dysesthesia and/or paresthesia (each scored on an 11-point NRS [0 = "none" to $10=$ "worst imaginable"]) and two questions that address the frequency and duration of pain.

\section{Statistical Analyses}

It was estimated that approximately $70 \%$ of the total study population would be eligible for inclusion in the per-protocol population. An estimated 125 patients in the per-protocol population would provide $80 \%$ power to differentiate between a responder rate 1 at week 6 of at least $60 \%$ and the null hypothesis responder rate 1 of less than $60 \%$, with a noninferiority margin of 14.3 . For responder rate 2 , it was estimated that 125 patients in the per-protocol population would provide $80 \%$ power to differentiate between a responder rate 2 at week 6 of at least $60 \%$ and the null hypothesis responder rate 2 of less than $60 \%$, with a noninferiority margin of $14.3 \%$. An estimated 125 patients in the per-protocol population would provide $80 \%$ power to reject the null hypothesis that the average pain intensity score at week -1 was not equivalent to that at week 6 (i.e., the difference in means was $\geq 0.673$ or farther away from 0 in the same direction), in favor of the alternative hypothesis 
that responder rate 1 was at least $60 \%$ (i.e., tapentadol PR provided noninferior analgesia relative to the previous WHO step III treatment). Given the rejection of the null hypothesis at the first two steps, it was estimated that a study population of 178 patients would provide $80 \%$ power to perform the described analyses of responder rate 1 , responder rate 2 , and pain intensity in a step-wise manner.

The primary endpoint (responder rate 1 at week 6 in the per-protocol population using the LOCF) was evaluated using a 1-sided Chi-square test, as were responder rate 2 at week 6 in the per-protocol population (LOCF) and responder rates 1 and 2 at weeks 6,8 , and 12 in the main analysis population (observed-case analysis). A 1-sided paired t-test was used to evaluate the changes from baseline in mean pain intensity (11-point NRS-3) at weeks 6, 8, and 12.

Weighted responses to each of the individual EQ-5D dimensions were used to derive an overall EQ-5D health status index score, with a possible score ranging from 0 ("dead") to 1 ("full health"). Weighted combinations of the 8 SF-36 subscale scores were used to calculate a physical component summary score and a mental component summary score; the possible score for each summary score ranged from 0 to 100 , with higher scores indicating better health. For the HADS, 7 of the 14 items were combined to yield an anxiety subscale score and the remaining seven items were combined to yield a depression subscale score; both the HADS anxiety and depression subscale scores had a possible score of 0 to 21 , with higher scores indicating worse symptoms of anxiety and depression, respectively. A one-sample paired t-test was used to analyze the changes from baseline to weeks 6,8 , and 12 in the EQ-5D health status index, the SF-36 subscale scores and summary scores, and the HADS anxiety and depression subscale scores.
The scores for the seven individual items on the painDETECT questionnaire addressing the frequency and quality of neuropathic pain symptoms and the total painDETECT score were summarized using descriptive statistics. The scores of the individual items on the NPSI were combined to yield the following subscores: burning pain, pressing pain, paroxysmal pain, evoked pain, and paresthesia/dysesthesia. For each NPSI subscore, the possible score ranged from $0-1$, with higher scores indicating more severe symptoms. The NPSI subscores were combined to yield an overall feeling NPSI score (possible score, $0-1$ ). The changes from baseline to weeks 6,8 , and 12 in the NPSI and painDETECT scores were analyzed using onesample paired t-tests. The neuropathic pain component to low back pain is challenging to assess [32] and may be underestimated by the painDETECT questionnaire; for this reason, the painDETECT unclear and positive subsets were combined for analyses of the NPSI, HADS, and individual painDETECT items presented herein.

For equianalgesic ratios of tapentadol to WHO step III opioids, the equipotent dose of tapentadol was defined as the mean TDD (average TDD during the 3 days prior to the visit) at which the pain intensity score was less than or equal to the pain intensity score at week -1 . The average TDD of a WHO step III analgesic taken during the 3 days prior to the baseline visit was used as the corresponding mean TDD for equianalgesia calculations. Equianalgesic ratios were calculated for tapentadol PR alone to PR formulations of WHO step III opioids alone and for tapentadol PR and tapentadol IR combined to PR and IR formulations of WHO step III opioids combined.

Separate analyses were performed for all effectiveness, function, and quality-of-life measures for the following two data sets: a data set that included results from weeks 9 
through 12 for patients who participated in the tapering substudy (the main data set) and a data set that excluded results from weeks 9 through 12 for patients who participated in the tapering substudy. Results of all effectiveness, function, and quality-of-life measures using the data set that excluded results from weeks 9 through 12 for patients who participated in the tapering substudy were evaluated using both observed-case analysis and LOCF for imputing missing assessments. WHO step I analgesics and coanalgesics were tapered in the substudy, which could potentially have resulted in pain peaks that might affect effectiveness, function, and quality-of-life analyses. In general, similar results were observed for the main data set (which included results from weeks 9 through 12 for patients who participated in the tapering substudy) and the data set that excluded results from weeks 9 through 12 for patients who participated in the tapering substudy. Unless otherwise specified, the results presented here are for the main data set (which included results from weeks 9 through 12 for patients who participated in the tapering substudy) using observed-case analysis. Results for the data set that excluded results from weeks 9 through 12 for patients who participated in the tapering substudy using observed-case analysis and LOCF are summarized in the Appendix.

\section{RESULTS}

\section{Patients}

This study was prematurely terminated due to slow recruitment and study drug shortages; as a result, the numbers of patients in the study populations were lower than initially planned. A total of 125 patients were included in the safety population, 123 patients were included in the main analysis population, and 94 patients were included in the per-protocol population. In the safety population, $37.6 \%(47 / 125)$ of patients were included in the painDETECT negative subset and $62.4 \%(78 / 125)$ of patients were included in the painDETECT unclear/positive subset (Table 1). Fifty-one patients in the painDETECT positive subset underwent additional diagnostic testing; based on this testing, 58.8\% (30/51) of these patients had a positive diagnosis of lumbar radiculopathy at screening or baseline.

Baseline and demographic characteristics for the overall safety population and by baseline painDETECT subset are summarized in Table 2, and the history of low back pain is summarized in Table 3. Patients in this study had a mean ongoing history of low back pain of 12.4 years. Thus, this patient population is representative of individuals with a long-term history of low back pain, with a history of

Table 1 Baseline painDETECT classifications ${ }^{\mathrm{a}}$

\begin{tabular}{lllll}
\hline $\begin{array}{l}\text { Baseline } \\
\text { painDETECT } \\
\text { classification, } n(\%)\end{array}$ & $\begin{array}{l}\text { Safety } \\
\text { population } \\
(\boldsymbol{n}=\mathbf{1 2 5})\end{array}$ & $\begin{array}{l}\text { Main analysis } \\
\text { population } \\
(\boldsymbol{n}=\mathbf{1 2 3})\end{array}$ & $\begin{array}{l}\text { Per-protocol } \\
\text { population } \\
(\boldsymbol{n}=\mathbf{9 4})\end{array}$ & $\begin{array}{l}\text { Tapering } \\
\text { substudy } \\
(\boldsymbol{n}=\mathbf{2 3})\end{array}$ \\
\hline Negative & $47(37.6)$ & $47(38.2)$ & $33(35.1)$ & $6(26.1)$ \\
Unclear & $26(20.8)$ & $25(20.3)$ & $19(20.2)$ & $6(26.1)$ \\
Positive & $52(41.6)$ & $51(41.5)$ & $42(44.7)$ & $11(47.8)$ \\
Unclear/positive & $78(62.4)$ & $76(61.8)$ & $61(64.9)$ & $17(73.9)$ \\
\hline
\end{tabular}

${ }^{a}$ Baseline painDETECT classifications were based on the total painDETECT score (possible score, 0-38); the likelihood of a neuropathic pain component to low back pain was classified as "negative" (score of 0-12), "unclear" (score of 13-18), or "positive" (score of 19-38) 
Table 2 Baseline and demographic characteristics (safety population)

\begin{tabular}{llll}
\hline Characteristic & $\begin{array}{l}\text { painDETECT } \\
\text { negative } \\
(n=47)\end{array}$ & $\begin{array}{l}\text { painDETECT } \\
\text { unclear/positive } \\
(n=78)\end{array}$ & $\begin{array}{l}\text { Total } \\
(n=125)\end{array}$ \\
\hline $\begin{array}{l}\text { Mean (SD) age, years } \\
\text { Gender, } n \text { (\%) }\end{array}$ & $59.9(11.57)$ & $55.3(11.99)$ & $57.1(12.00)$ \\
$\quad$ Female & $30(63.8)$ & $46(59.0)$ & $76(60.8)$ \\
$\quad$ Male & $17(36.2)$ & $32(41.0)$ & $49(39.2)$ \\
Mean (SD) BMI, kg/m $\mathrm{m}^{2}$ & $28.6(4.84)$ & $28.2(5.53)$ & $28.3(5.27)$ \\
Race, $n(\%)$ & $47(100.0)$ & $77(98.7)$ & $124(99.2)$ \\
$\quad$ White & 0 & $1(1.3)$ & $1(0.8)$ \\
$\quad$ Asian & & &
\end{tabular}

$B M I$ body mass index, $S D$ standard deviation

Table 3 History of low back pain (safety population)

\begin{tabular}{llll}
\hline Parameter & $\begin{array}{l}\text { painDETECT } \\
\text { negative } \\
(n=47)\end{array}$ & $\begin{array}{l}\text { painDETECT } \\
\text { unclear/positive } \\
(n=77)\end{array}$ & $\begin{array}{l}\text { Total } \\
(n=124)\end{array}$ \\
\hline $\begin{array}{l}\text { Mean (SD) duration of pain, years } \\
\text { Mean (SD) time to first pain-related } \\
\text { consultation, months }\end{array}$ & $13.8(11.30)$ & $11.5(10.91)$ & $12.4(11.07)$ \\
$\begin{array}{l}\text { Mean (SD) number of doctors visited } \\
\text { since pain started }\end{array}$ & $19.1(44.79)$ & $6.6(23.92)$ & $11.3(33.78)$ \\
$\begin{array}{l}\text { Mean (SD) number of consultations } \\
\text { within 3 months }\end{array}$ & $4.8(3.14)$ & $5.8(5.49)$ & $5.4(4.75)$ \\
$\begin{array}{l}\text { Hospitalization due to pain, } n \text { (\%) } \\
\text { Mean (SD) number of analgesic regimens }\end{array}$ & $2.5(1.91)$ & $3.3(5.20)$ & $3.0(4.27)$ \\
$\begin{array}{l}\text { since pain started } \\
\text { Mean }(S D) \text { times of being off work due }_{\text {to pain per year }}\end{array}$ & $5.3(3.61)$ & $54(69.2)$ & $77(61.6)$ \\
\hline
\end{tabular}

$S D$ standard deviation

${ }^{a}$ painDETECT negative, $n=46$; painDETECT unclear/positive, $n=76$; total, $n=122$

${ }^{\mathrm{b}}$ painDETECT negative, $n=9$; painDETECT unclear/positive, $n=14$; total, $n=23$

multiple previous analgesic regimens and a high degree of suffering, particularly for those with a neuropathic pain component.

During week -1 , all but two patients took WHO step III analgesics. For the two patients who did not receive WHO step III pretreatment, the absence of pretreatment was detected only after the patients had enrolled and entered the study; both patients were considered major protocol violators. The most commonly used WHO step III analgesics during week -1 included oral oxycodone, transdermal fentanyl, transdermal or IR (sublingual) buprenorphine, and oral morphine (Table 4). A total of $21.6 \%$ $(27 / 125)$ of patients were taking WHO step II analgesics during week -1 . The WHO step II 
Table 4 WHO Step III analgesics taken during week -1 (safety population)

\begin{tabular}{ll}
\hline WHO step III analgesic, $n(\%)^{\mathrm{a}}$ & $\begin{array}{l}\text { Total } \\
(\boldsymbol{n}=125)^{\mathrm{b}}\end{array}$ \\
\hline Oxycodone & $40(32.0)$ \\
Fentanyl & $32(25.6)$ \\
Buprenorphine & $27(21.6)$ \\
Morphine & $20(16.0)$ \\
Hydromorphone & $10(8.0)$ \\
Naloxone $\mathrm{HCl}$, oxycodone $\mathrm{HCl}$ & $2(1.6)$ \\
Levomethadone & $1(0.8)$ \\
Methadone $\mathrm{HCl}$ & $1(0.8)$ \\
\hline
\end{tabular}

HCl hydrochloride, WHO World Health Organization ${ }^{a}$ Fentanyl was administered transdermally. Buprenorphine was administered transdermally or as an immediate-release formulation (sublingually). All other WHO step III opioids were administered orally

${ }^{\mathrm{b}}$ Two patients took no WHO step III analgesics during week -1

analgesics taken concomitantly during week -1 were tramadol (17.6\% [22/125]); a fixed-dose combination of tramadol/paracetamol (2.4\% [3/125]); a fixed-dose combination of codeine/paracetamol (0.8\% [1/125]); and a fixeddose combination of tilidine $\mathrm{HCl} /$ naloxone $\mathrm{HCl}(0.8 \%$ [1/125]). The TDDs of WHO step III opioids that patients were taking at baseline were converted to MEDs, which are summarized in Table 5. The starting doses of tapentadol for patients taking TDDs of WHO step III opioids in all three MED cohorts ( $\leq 100 \mathrm{mg}$ /day, 101-160 mg/day, >160 mg/day) were generally lower than the MEDs (in some cases, more than 50\% lower). Week -1 MED cohort data were not available for five patients in the safety population; those five patients did not have proper documentation of analgesic medication in their diaries, which was used to determine starting doses, so the starting doses for those patients were determined by the investigator. Three of those patients took starting doses of tapentadol PR of $100 \mathrm{mg}$ b.i.d. (corresponding
Table 5 MEDs of TDDs of previous WHO step III opioids at baseline (safety population)

\begin{tabular}{ll}
\hline Average MED ${ }^{\mathrm{a}}, n(\%)$ & $\begin{array}{l}\text { Total } \\
(n=120)^{\mathrm{b}}\end{array}$ \\
\hline$\leq 100 \mathrm{mg} /$ day & $68(56.7)$ \\
$101-160 \mathrm{mg} /$ day & $29(24.2)$ \\
$>160 \mathrm{mg} /$ day & $23(19.2)$ \\
\hline
\end{tabular}

$M E D$ morphine equivalent dose, $T D D$ total daily dose, WHO World Health Organization

${ }^{a}$ Includes all formulations of all opioids taken

b The MED cohort was unknown for five patients in the safety population

to the MED cohort of 101-160 mg/day), one patient took a starting dose of tapentadol PR of $50 \mathrm{mg}$ b.i.d. (corresponding to the MED cohort of $\leq 100 \mathrm{mg}$ /day), and one patient received study medication but was lost to follow-up.

During the study, $83.2 \%(104 / 125)$ of patients took concomitant analgesics or coanalgesics. A total of $63.2 \%(79 / 125)$ of patients took concomitant WHO step I analgesics; the most commonly used WHO step I analgesics during the study included paracetamol (22.4\% [28/125]), meloxicam (11.2\% [14/125]), metamizole (dipyrone; $11.2 \%$ [14/125]), diclofenac $(9.6 \%$ [12/125]), and ibuprofen (8.8\% [11/125]). Concomitant coanalgesics were used by $54.4 \%$ $(68 / 125)$ of patients during the study, and the most commonly used coanalgesics during the study included pregabalin (17.6\% [22/125]), amitriptyline (12.8\% [16/125]), gabapentin (10.4\% [13/125]), and clonazepam (8.8\% [11/125]). The use of WHO step II and WHO step III analgesics (which were prohibited according to the study protocol) was reported for $3.2 \%(4 / 125)$ and $5.6 \%(7 / 125)$ of patients, respectively; two of the patients who took WHO step II or III analgesics were excluded from the per-protocol population due to major protocol deviations and one of the patients who took WHO step III analgesics discontinued the study at visit 6 . 
Overall, 25.6\% (32/125) of patients discontinued the study prematurely. The reasons for premature study discontinuation included adverse events (16.0\% [20/125]), withdrawal of consent for any reason $(4.0 \%$ [5/125]), a lack of efficacy $(4.0 \%$ [5/125]), study medication noncompliance $(0.8 \%[1 / 125])$, or unknown reasons $(0.8 \%$ [1/125]). The one patient who discontinued because of safety reasons not related to the study medication had a serious TEAE of myocardial infarction that was considered by the investigator to be not related to treatment with tapentadol.

\section{Effectiveness, Function, and Quality of Life}

Unless otherwise specified, effectiveness, function, and quality-of-life results presented here are for the main data set (which included results from weeks 9 through 12 for patients who participated in the tapering substudy) using observed-case analysis. For the primary endpoint, responder rate 1 (LOCF) in the perprotocol population was $80.9 \%(76 / 94)$ at week 6 and was significantly different from the null hypothesis responder rate of less than $60 \%$ $(P<0.0001)$, indicating that the effectiveness of tapentadol PR was noninferior to previous WHO step III opioids. Responder rate 2 (LOCF) in the per-protocol population was $66.0 \%(62 / 94)$ at week 6 and was significantly different from the null hypothesis responder rate of less than $60 \%$ $(P<0.0001)$. In the main analysis population, responder rate 1 increased from baseline to week 4 (78.6\% [81/103]) and remained relatively steady during the remainder of the study; responder rate 1 was $80.4 \%(82 / 102)$ at week $6,82.3 \%$ (79/96) at week 8, and 81.7\% (76/93) at week 12. For patients in the painDETECT negative and unclear/positive subsets, respectively, responder rate 1 was $81.1 \%(30 / 37)$ and $80.0 \%(52 / 65)$ at week $6,94.1 \%(32 / 34)$ and $75.8 \%(47 / 62)$ at week 8 , and $91.2 \%(31 / 34)$ and $76.3 \%(45 / 59)$ at week 12 . Responder rate 2 in the main analysis population was $63.7 \%(65 / 102)$ at week $6,74.0 \%$ (71/96) at week 8, and 74.2\% (69/93) at week 12. For patients in the painDETECT negative and unclear/positive subsets, respectively, responder rate 2 was $62.2 \%(23 / 37)$ and $64.6 \%(42 / 65)$ at week $6,79.4 \%(27 / 34)$ and $71.0 \%(44 / 62)$ at week 8 , and $73.5 \%(25 / 34)$ and $74.6 \%(44 / 59)$ at week 12 . The mean (SD) pain intensity score at baseline was $4.8(0.75)$; pain relief was maintained during the initial switch to tapentadol PR. Significant decreases in mean (SD) pain intensity scores were observed with continued tapentadol PR treatment, with a change of -0.9 (1.89) by week $6,-1.1(1.91)$ by week 8 , and $-1.3(2.10)$ by week $12(P<0.0001$ for the change from baseline for all comparisons; Fig. 1). Similar decreases in pain intensity were reported for patients diagnosed with lumbar radiculopathy $(n=30)$; mean $(\mathrm{SD})$ pain intensity for pain radiating towards or into the leg was 4.9 (1.31) at baseline and decreased by $-1.4(2.31)$ by week $6,-1.5$ (2.58) by week 8 , and $-1.2(2.59)$ by week 12 .

At baseline, none of the patients in the main analysis population rated their satisfaction with their previous WHO step III analgesic treatment as "good," "very good," or "excellent." The percentage of patients rating their satisfaction with tapentadol PR treatment as "good," "very good," or "excellent" was 72.5\% (74/102) at week 6 and 82.8\% (77/93) at week 12 (Fig. 2). On the PGIC, the percentage of patients who reported that their overall status was "minimally improved," "much improved," or "very much improved" since starting study treatment was $79.4 \%(81 / 102)$ at week $6,83.3 \%(80 / 96)$ at week 8, and $87.1 \%(81 / 93)$ at week 12 (Fig. 3). On the CGIC, a rating of "minimally improved," "much improved," or "very much improved" for the patients' overall status since starting study 


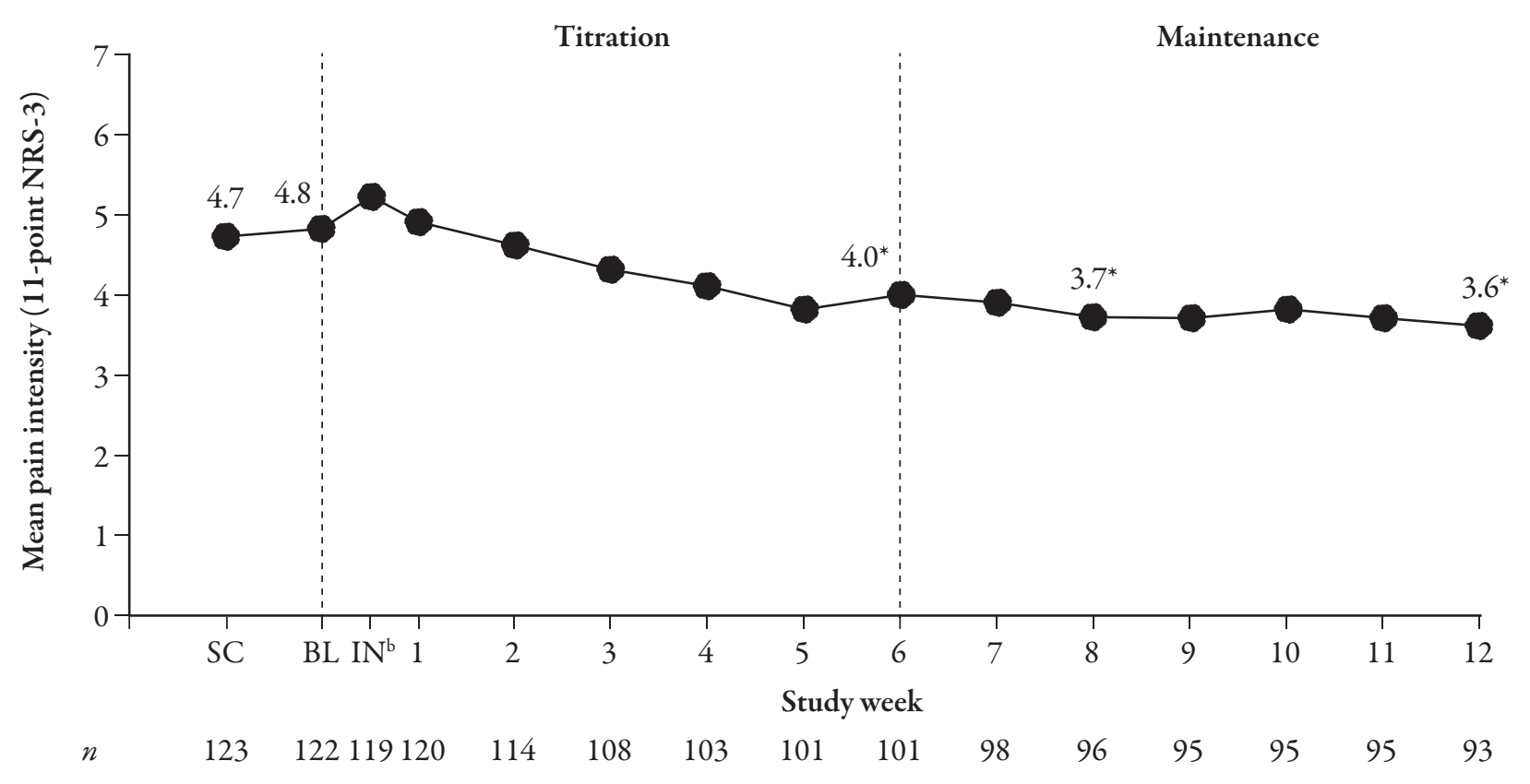

Fig. 1 Mean pain intensity (NRS-3) over time (main analysis population; observed-case analysis). ${ }^{a} B L$ baseline, $I N$ interim, NRS-3 numerical rating scale-3, $S C$ screening, $S D$ standard deviation, $W$ week. ${ }^{a} S D$ : SC, $0.80 ; \mathrm{BL}, 0.75$; W1, 1.68; W2, 1.63; W3, 1.51; W4, 1.56; W5, 1.69; W6, 1.77; W7, 1.82; W8, 1.86; W9, 1.89; W10, 1.90; W11, 1.89; W12, 1.97.

${ }^{\mathrm{b}}$ The interim visit occurred 3 to 4 days after the baseline visit. ${ }^{*} P<0.0001$ for the change from baseline

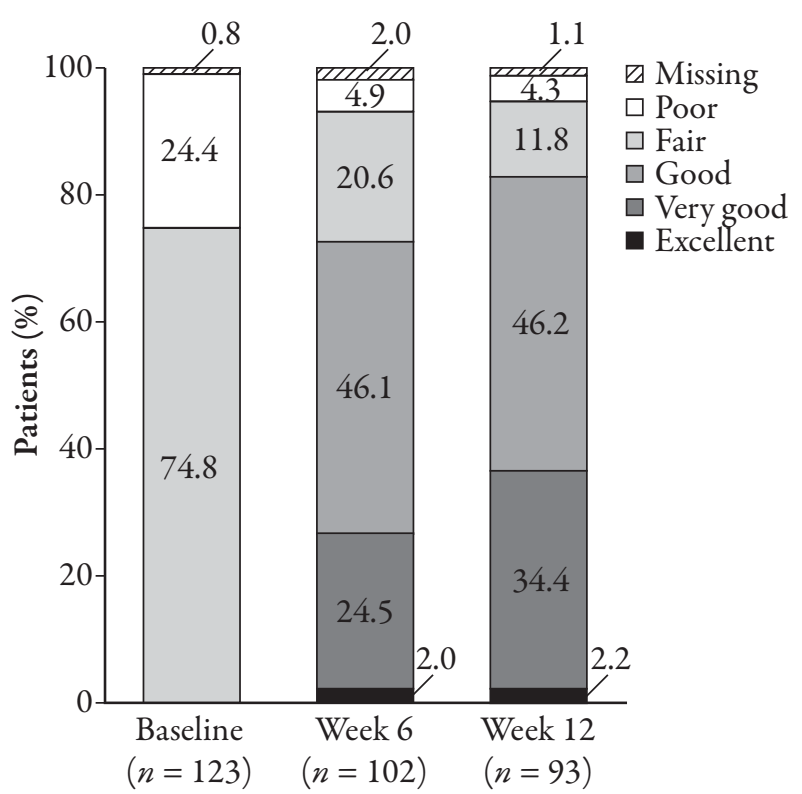

Fig. 2 Subject satisfaction with treatment ratings at baseline, week 6, and week 12 (main analysis population; observed-case analysis). a a Percentages may not total $100.0 \%$ because of rounding

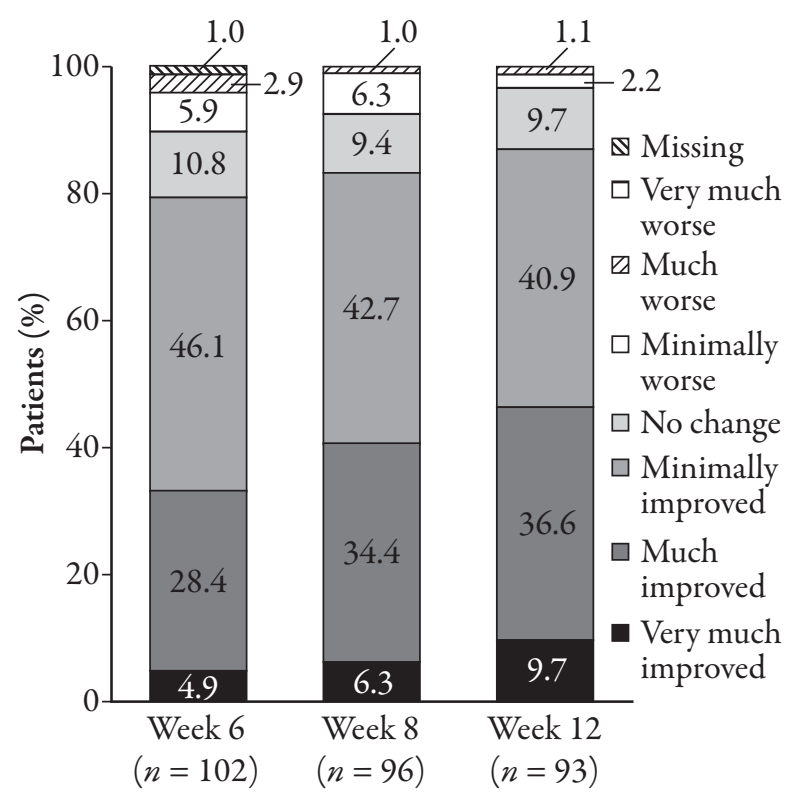

Fig. 3 PGIC ratings at weeks 6, 8, and 12 (main analysis population; observed-case analysis). ${ }^{a}$ PGIC patient global impression of change. a Percentages may not total 100.0\% because of rounding 
treatment was reported by $80.4 \%(82 / 102)$ of investigators at week $6,84.4 \%(81 / 96)$ of investigators at week 8 , and $87.1 \%(81 / 93)$ of investigators at week 12 .

Significant increases were observed in the mean EQ-5D health status index score from baseline to week 6 (mean [SD] change from baseline, 0.15 [0.266]), from baseline to week $8(0.16[0.294])$, and from baseline to week 12 (0.16 [0.257]; $P<0.0001$ for all comparisons). At week 6, significant increases from baseline were observed in all mean SF-36 domain scores $(P<0.05$ for all comparisons), except for the role emotional score (Fig. 4). Significant increases from baseline were observed in all mean SF-36 domain scores at week 12 ( $P<0.05$ for all comparisons; Fig. 4). The mean physical component summary score increased significantly from baseline to week 6 (mean [SD] change from baseline, 4.3 [7.88]) and week 12 (3.8 [7.91]; $P<0.0001$ for both comparisons). The mean (SD) mental component summary score increased significantly from baseline to week 12 (mean [SD] change from baseline, 5.1 [10.62]; $P<0.0001)$, but not from baseline to week 6 (1.6 [10.53]).

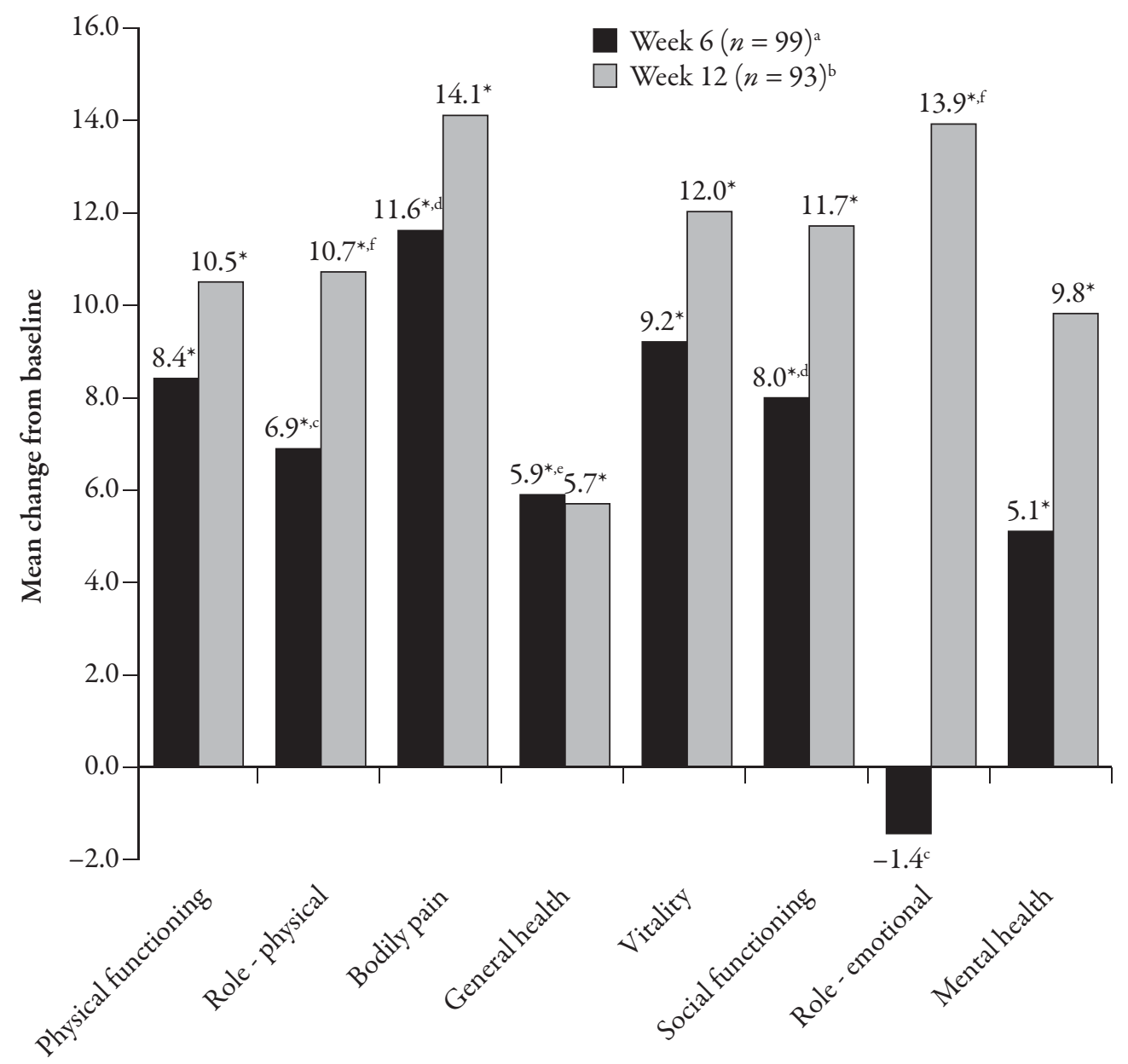

Fig. 4 Mean changes in SF-36 domain scores from baseline to week 6 and week 12 (main analysis population; observed-case analysis). $S D$ standard deviation, $S F-36$ Short Form-36. ${ }^{a} S D$ : Physical functioning, 18.65; role - physical, 28.89; bodily pain, 20.00; general health, 15.10; vitality, 17.44; social functioning, 24.47; role - emotional, 42.26; mental health, 16.73 .

${ }^{b} S D$ : Physical functioning, 19.96; role - physical, 31.65; bodily pain, 22.84; general health, 14.98; vitality, 21.03; social functioning, 27.07; role - emotional, 43.06; mental health, $17.70 .{ }^{\mathrm{c}} n=98 .{ }^{\mathrm{d}} n=101 .{ }^{\mathrm{e}} n=100 .{ }^{\mathrm{f}} n=91 .{ }^{*} P<0.05$ 
In the main analysis population, the mean (SD) HADS anxiety subscale score was 7.5 (4.23) at baseline and decreased significantly from baseline to weeks 6,8 , and $12(P<0.005$ for all comparisons; Fig. 5a). Significant decreases were also observed in the mean (SD) HADS depression subscale score from baseline $(7.9$ [4.54]) to weeks 6,8 , and $12(P<0.005$ for all comparisons; Fig. 5b). At baseline, the mean (SD) HADS anxiety and depression subscale scores were higher (and clinically relevant) for patients in the painDETECT unclear/positive group (8.5 [3.96] and 8.9 [4.33], respectively; scores of $\geq 8$ are considered to indicate the likely presence of anxiety or depression [29]) than for patients in the painDETECT negative group (5.9 [4.21] and 6.2 [4.40], respectively; not in line with a clinically relevant score). For patients in the

a) Anxiety

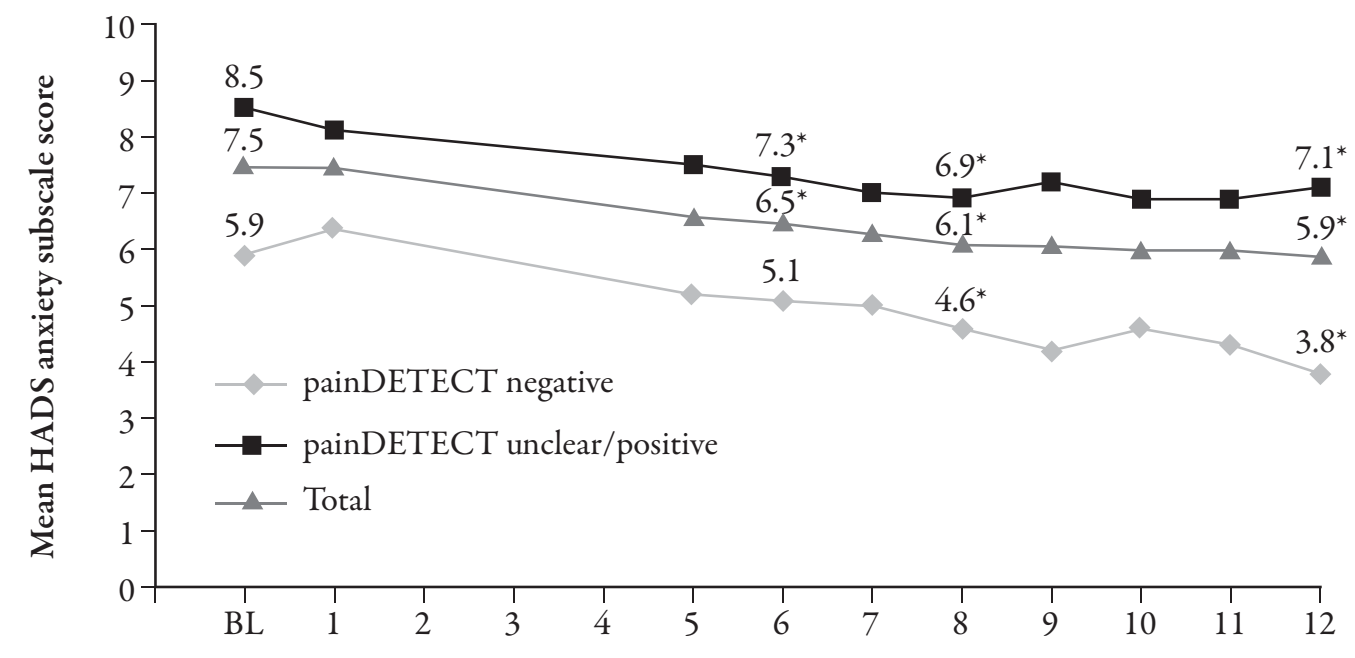

Study week

\begin{tabular}{|l|l|l|l|l|l|l|l|l|l|l|l|l|l|}
\hline $\begin{array}{l}\text { painDETECT } \\
\text { negative, } n\end{array}$ & 45 & 44 & & & & 37 & 35 & 35 & 34 & 32 & 33 & 33 & 34 \\
\hline $\begin{array}{l}\text { painDETECT } \\
\text { unclear/positive, } n\end{array}$ & 73 & 73 & & & & 64 & 64 & 64 & 62 & 58 & 57 & 57 & 59 \\
\hline \begin{tabular}{l} 
Total, $n$ \\
\hline
\end{tabular} & 118 & 117 & & & & 101 & 99 & 99 & 96 & 90 & 90 & 90 & 93 \\
\hline
\end{tabular}

Fig. 5 Mean HADS (a) anxiety and (b) depression subscale scores for the overall population and by painDETECT subset (observed-case analysis; main analysis population) (continued on next page). BL baseline, HADS Hospital Anxiety and Depression Scale, $S D$ standard deviation, $W$ week. ${ }^{2} S D$ : painDETECT negative: BL, 4.21; W 1, 3.93; W5, 3.82; W6, 3.69; W7, 3.43; W8, 3.40; W9, 3.43; W 10, 3.57; W11, 3.12; W12, 3.26; painDETECT unclear/positive: BL, 3.96; W 1, 4.22; W5, 3.71; W6, 4.02; W7, 3.82; W8, 4.16; W9, 4.16; W10, 4.30; W11, 4.08; W12, 4.01; total: BL, 4.23; W 1, 4.18; W5,

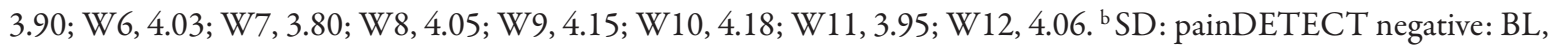
4.40; W1, 4.70; W5, 3.65; W6, 3.46; W7, 3.49; W8, 3.62; W9, 3.66; W10, 3.76; W11, 3.98; W12, 3.42; painDETECT unclear/positive: BL, 4.33; W1 4.18; W5, 3.82; W6, 4.14; W7, 4.04; W8, 4.53; W9, 4.61; W10, 4.92; W11, 4.46; W12, 4.67; total: BL, 4.54; W1, 4.46; W5, 3.99; W6, 4.17; W7, 4.02; W8, 4.42; W9, 4.54; W10, 4.73; W11, 4.49; W12, 4.57. ${ }^{*} P<0.05$ for the change from baseline 
b) Depression $^{\text {b }}$

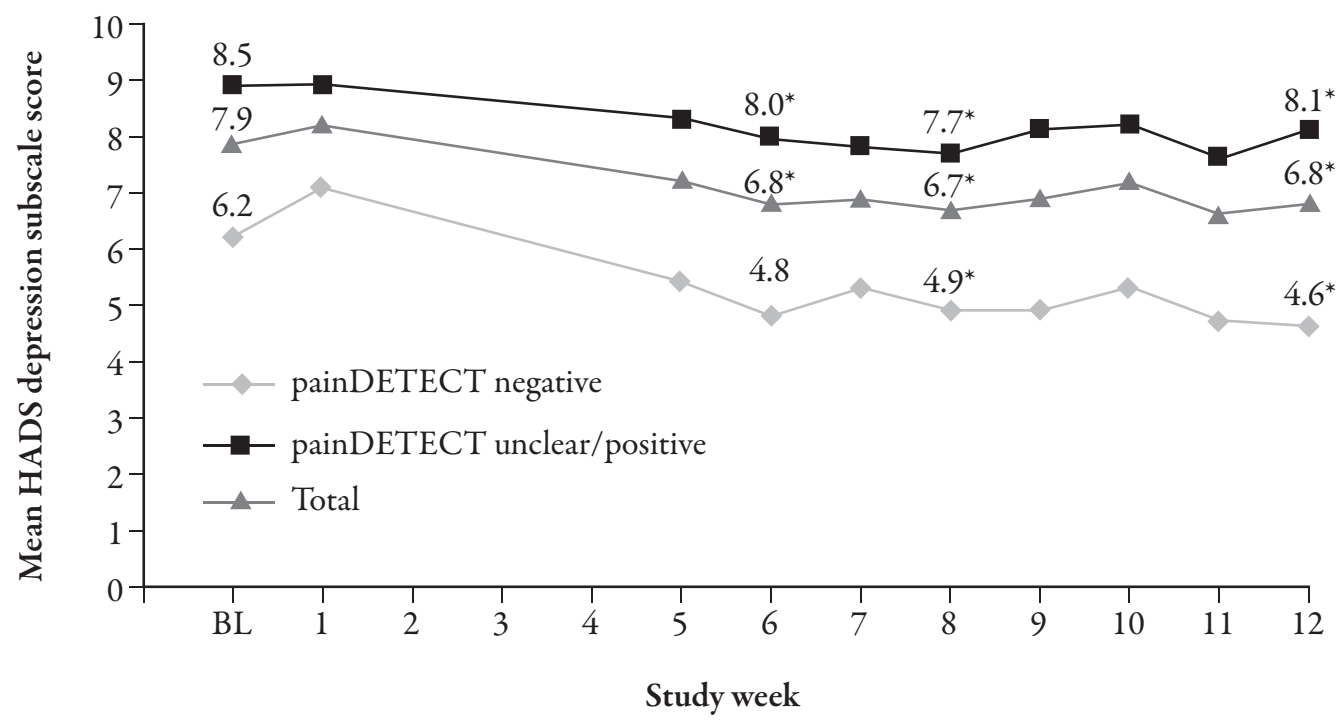

\begin{tabular}{|l|l|l|l|l|l|l|l|l|l|l|l|l|l|}
\hline $\begin{array}{l}\text { painDETECT } \\
\text { negative, } n\end{array}$ & 45 & 44 & & & & 37 & 35 & 35 & 34 & 32 & 33 & 33 & 34 \\
\hline $\begin{array}{l}\text { painDETECT } \\
\text { unclear/positive, } n\end{array}$ & 73 & 73 & & & & 64 & 64 & 64 & 62 & 58 & 57 & 57 & 59 \\
\hline \begin{tabular}{l} 
Total, $n$ \\
\hline
\end{tabular} & 118 & 117 & & & & 101 & 99 & 99 & 96 & 90 & 90 & 90 & 93 \\
\hline
\end{tabular}

Fig. 5 continued

painDETECT unclear/positive group, significant decreases from baseline were observed in the mean HADS anxiety subscale score at weeks 6 , 8 , and 12 ( $P<0.001$ for all comparisons) and in the mean HADS depression subscale score at weeks 6 and 8 ( $P<0.01$ for both comparisons; Fig. 5). For patients in the painDETECT negative group, significant decreases from baseline were observed in the mean HADS anxiety and depression subscale scores at weeks 8 and 12 ( $P<0.05$ for all comparisons; Fig. 5), but not at week 6.

Responder rates, mean pain intensity scores, subject satisfaction with treatment ratings, PGIC and CGIC ratings, EQ-5D health status index scores, SF-36 domain and summary scores, and
HADS anxiety and depression subscale scores were comparable whether results from weeks 9 through 12 were included or excluded for patients who participated in the tapering substudy (Appendix Tables A1-A7). Results for these effectiveness, function, and quality-of-life measures for the data set that excluded results from weeks 9 through 12 for patients who participated in the tapering substudy and using LOCF are also presented in Appendix Tables A1-A7.

\section{Treatment Exposure and Equianalgesia to Prior WHO Step III Opioids}

Table 6 shows the number of dose adjustments required to reach the minimum target of 
Table 6 Number of dose adjustments to reach the minimum target of titration at visit 6 (main analysis population) in responders and the overall population (responders/nonresponders) by painDETECT category ${ }^{a}$

\begin{tabular}{lllll}
\hline $\begin{array}{l}\text { Number of dose } \\
\text { adjustments, } n(\%)\end{array}$ & $\begin{array}{l}\text { Responders }^{\mathrm{b}} \\
(n=94)\end{array}$ & Overall population & & \\
\cline { 3 - 5 } & & $\begin{array}{l}\text { painDETECT } \\
\text { negative }(n=47)\end{array}$ & $\begin{array}{l}\text { painDETECT } \\
\text { unclear/positive }(n=76)\end{array}$ & $\begin{array}{c}\text { Total } \\
(n=123)\end{array}$ \\
\hline 0 & $70(74.5)$ & $31(66.0)$ & $49(64.5)$ & $80(65.0)$ \\
1 & $13(13.8)$ & $8(17.0)$ & $12(15.8)$ & $20(16.3)$ \\
2 & $7(7.4)$ & $6(12.8)$ & $7(9.2)$ & $13(10.6)$ \\
3 & $3(3.2)$ & $2(4.3)$ & $7(9.2)$ & $9(7.3)$ \\
4 & $1(1.1)$ & 0 & $1(1.3)$ & $1(0.8)$ \\
\hline
\end{tabular}

${ }^{a}$ The minimum target of titration was defined as achieving at least similar efficacy compared to the previous treatment (i.e., pain intensity score [11-point numerical rating scale-3] that was the same or lower than at baseline)

${ }^{\mathrm{b}}$ Based on the primary endpoint responder rate 1 analysis

titration at visit 6 by responder status and by painDETECT subset. In the main analysis population, approximately two-thirds of patients required no dose adjustment to reach the minimum target of titration (i.e., pain intensity score [11-point NRS-3] that was the same or lower than at baseline) by visit 6 . For responders (based on the primary endpoint responder rate 1 analysis), no tapentadol PR dose adjustment was required by approximately three-quarters of patients to reach the minimum target of titration by visit 6 . The mean (SD) TDD of tapentadol PR at week 6 (after doses had stabilized) was 322.8 (120.73) $\mathrm{mg}$, and the mean (SD) TDD of tapentadol IR at week 6 was 24.6 (32.96) $\mathrm{mg}$. The dose distribution of tapentadol PR at week 6 is described in Table 7. Equianalgesic ratios for tapentadol PR alone to PR formulations of oral oxycodone, transdermal buprenorphine, transdermal fentanyl, oral morphine, and oral hydromorphone are summarized in Table 8, along with equianalgesic ratios for tapentadol PR plus tapentadol IR to PR and IR formulations of the same five WHO step III opioids (oral oxycodone, transdermal [PR] and oral [sublingual; IR] buprenorphine, transdermal fentanyl, oral morphine, and oral hydromorphone). In the higher MED dose ranges
Table 7 Doses of tapentadol PR and tapentadol IR at week 6 (main analysis population)

\begin{tabular}{cl}
\hline Dose, $n(\%)$ & $\begin{array}{l}\text { Total } \\
(n=101)\end{array}$ \\
\hline Tapentadol PR & \\
50 mg b.i.d. & $9(8.9)$ \\
100 mg b.i.d. & $22(21.8)$ \\
150 mg b.i.d. & $22(21.8)$ \\
200 mg b.i.d. & $33(32.7)$ \\
250 mg b.i.d. & $15(14.9)$ \\
Tapentadol IR & \\
None & $55(54.5)$ \\
$>0$ to < 50 mg & $14(13.9)$ \\
50 mg & $19(18.8)$ \\
$>50$ to $<100$ mg & $3(3.0)$ \\
100 mg & $10(9.9)$ \\
\hline
\end{tabular}

b.i.d. twice daily, $I R$ immediate release, $P R$ prolonged release

(particularly beyond the equianalgesic dose ratio of the maximum daily dose [500 mg/day] of tapentadol), equianalgesic ratios decreased in favor of tapentadol (Table 9).

\section{Neuropathic Pain Component}

Significant decreases from baseline were shown in the mean (SD) total painDETECT score at weeks 6,8 , and 12 in the painDETECT 
Table 8 Equianalgesic ratios of tapentadol to WHO step III opioids (main analysis population)a

\begin{tabular}{llll}
\hline WHO step III opioid $^{\text {a }}$ & $n$ & PR formulations & PR and IR formulations \\
\hline Oxycodone & $35^{\mathrm{b}}$ & $4.3: 1$ & $5.3: 1$ \\
Buprenorphine & $24^{\mathrm{c}}$ & $170.4: 1$ & $210.0: 1$ \\
Fentanyl & 22 & $223.9: 1$ & $250.7: 1$ \\
Morphine & 14 & $2.9: 1$ & $3.0: 1$ \\
Hydromorphone & 8 & $8.3: 1$ & $10.5: 1$ \\
\hline
\end{tabular}

$I R$ immediate release, $P R$ prolonged release, $W H O$ World Health Organization

${ }^{a}$ Fentanyl was administered transdermally. Buprenorphine was administered transdermally or as an IR formulation (sublingually). All other WHO step III opioids were administered orally

${ }^{\mathrm{b}} \mathrm{PR}$ formulations, $n=34$

${ }^{\mathrm{c}} \mathrm{PR}$ formulations, $n=21$

Table 9 Equianalgesic ratios of tapentadol to prior WHO step III opioids by MED of prior therapy (main analysis population)

\begin{tabular}{llll}
\hline Average MED & $n$ & PR formulations & PR and IR formulations \\
\hline$\leq 100 \mathrm{mg} /$ day & $60^{\mathrm{a}}$ & $3.0: 1$ & $3.6: 1$ \\
$101-160 \mathrm{mg} /$ day & 27 & $1.9: 1$ & $2.3: 1$ \\
$>160 \mathrm{mg} /$ day & 18 & $1.4: 1$ & $1.6: 1$ \\
\hline
\end{tabular}

$I R$ immediate release, $M E D$ morphine equivalent dose, $P R$ prolonged release, $W H O$ World Health Organization

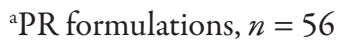

unclear and positive subsets $(P<0.005$ for all comparisons; Fig. 6). Patients with a painDETECT positive score at baseline had an unclear score by week 6 and for the remainder of the study, and patients with a painDETECT unclear score at baseline had a negative score by week 6 and for the remainder of the study. In the baseline painDETECT unclear/positive subset, significant improvements from baseline to week 6 and from baseline to week 12 were observed in all individual painDETECT item scores $(P<0.05$ for all comparisons; Table 10), and the NPSI paroxysmal pain, pressing pain, evoked pain, burning pain, and paresthesia/dysesthesia subscores and the NPSI overall feeling score improved significantly from baseline to week 6 and from baseline to week $12(P<0.05$ for all comparisons; Table 11a). On the NPSI, the number of pain attacks that patients in the painDETECT unclear/positive subset reported in a 24 -h period (Table 11b) decreased over the course of the study.

Similar mean NPSI scores, numbers of pain attacks in a $24-\mathrm{h}$ period (on the NPSI), and amounts of time that patients reported spontaneous pain in a 24 -h period (on the NPSI) were observed at week 12 whether results from weeks 9 through 12 for patients who participated in the tapering substudy were included or excluded (Appendix Table A8). Improvements in NPSI results were consistent at weeks 6 and 12 whether LOCF or no imputation method was used (Appendix Table A8).

\section{Tapering Substudy}

Overall, 87.0\% (20/23) of patients reduced their dose of WHO step I analgesics or coanalgesics in the course of the tapering substudy, and $65.2 \%(15 / 23)$ of patients completely stopped 


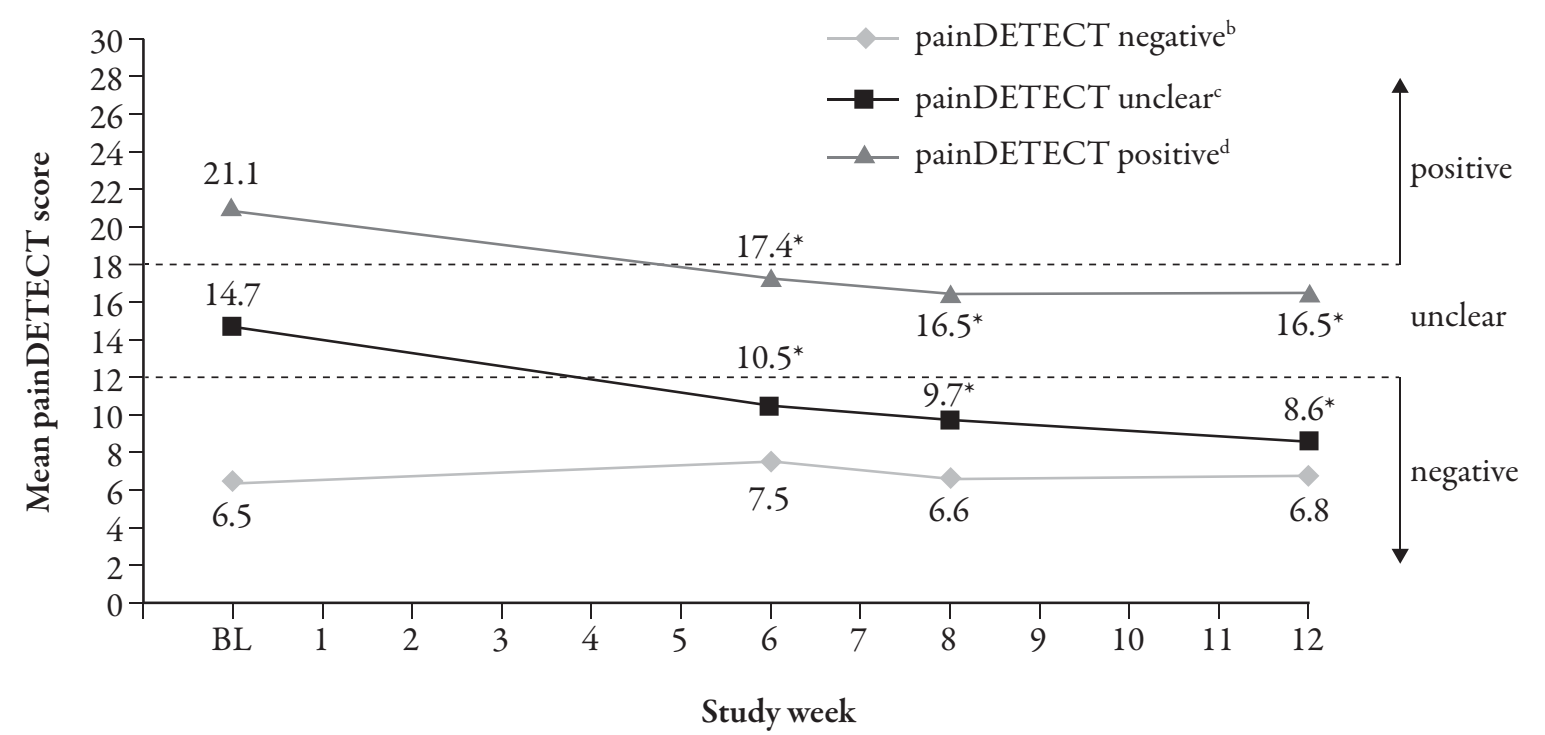

Fig. 6 Mean painDETECT scores over time by baseline painDETECT subset (observed-case analysis; main analysis population). ${ }^{a} B L$ baseline, $S D$ standard deviation, $W$ week. ${ }^{2}$ SD: painDETECT negative: $\mathrm{BL}, 3.90$; W6, 4.18; W8, 4.20; W12, 4.85; painDETECT unclear: BL, 2.39; W6, 4.75; W8, 5.52; W12, 5.43; painDETECT positive: BL, 3.39; W6, 5.95; $\mathrm{W} 8,6.63 ; \mathrm{W} 12,7.17 .{ }^{\mathrm{b}} \mathrm{BL}, n=44 ; \mathrm{W} 6, n=35 ; \mathrm{W} 12, n=33 .{ }^{\mathrm{c}} \mathrm{BL}, n=24 ; \mathrm{W} 6, n=20 ; \mathrm{W} 12, n=19 .{ }^{\mathrm{d}} \mathrm{BL}, n=50$; W6, $n=45 ; \mathrm{W} 12, n=40 .{ }^{*} P<0.005$ for the change from baseline

Table 10 Mean (SD) painDETECT individual item scores in the baseline painDETECT unclear/positive subsets (observed-case analysis; main analysis population)

\begin{tabular}{llll}
\hline Mean (SD) painDETECT score & $\begin{array}{l}\text { Baseline } \\
(n=74)\end{array}$ & $\begin{array}{l}\text { Week 6 } \\
(n=65)\end{array}$ & $\begin{array}{l}\text { Week 12 } \\
(n=59)\end{array}$ \\
\hline Burning sensation & $2.5(1.40)$ & $2.0(1.49)^{*}$ & $1.8(1.44)^{*}$ \\
Tingling or prickling sensation & $2.9(1.08)$ & $2.2(1.33)^{*}$ & $2.1(1.39)^{*}$ \\
Light touching painful & $1.7(1.18)$ & $1.2(1.21)^{*}$ & $1.0(1.31)^{*}$ \\
Sudden pain attacks & $3.1(1.11)$ & $2.0(1.36)^{*}$ & $1.9(1.57)^{*}$ \\
Cold or heat occasionally painful & $1.9(1.30)$ & $1.5(1.47)^{*}$ & $1.5(1.45)^{*}$ \\
Sensation of numbness & $2.8(1.11)$ & $2.4(1.04)^{*}$ & $2.2(1.26)^{*}$ \\
Slight pressure triggers pain & $2.8(1.21)$ & $2.5(1.47)^{*}$ & $2.1(1.51)^{*}$ \\
\hline
\end{tabular}

$S D$ standard deviation

${ }^{*} P<0.05$ for the change from baseline, indicating improvement in symptoms

their WHO step I analgesic or coanalgesic doses (Table 12$)$. A total of $82.4 \%(14 / 17)$ of patients reduced their dose of coanalgesics during the substudy, and $65.2 \%(15 / 23)$ of patients completely stopped their WHO step I analgesic or coanalgesic doses (Table 12). All patients $(n=6)$ who were tapering their dose of WHO step I analgesics during the substudy completely stopped their WHO step I analgesic doses (Table 12). The WHO step I analgesics reduced in the substudy included diclofenac sodium, ketoprofen, and paracetamol; the coanalgesics reduced in substudy A included amitriptyline, duloxetine, gabapentin, pregabalin, tolperisone, 
Table 11 NPSI results in the baseline painDETECT unclear/positive subset: (a) mean (SD) NPSI overall feeling and subscores, (b) total number of pain attacks within the past $24 \mathrm{~h}$ (observed-case analysis; main analysis population)

\begin{tabular}{llll}
\hline a) Overall feeling and subscores & & & \\
\hline Mean (SD) NPSI score & $\begin{array}{l}\text { Baseline } \\
(n=71)\end{array}$ & $\begin{array}{l}\text { Week 6 } \\
(n=61)\end{array}$ & $\begin{array}{l}\text { Week 12 } \\
(n=56)\end{array}$ \\
\hline Overall feeling score & $0.41(0.158)^{\mathrm{a}}$ & $0.30(0.178)^{\mathrm{b}, *}$ & $0.28(0.211)^{*}$ \\
Burning pain subscore & $0.41(0.284)$ & $0.32(0.273)^{*}$ & $0.27(0.281)^{*}$ \\
Pressing pain subscore & $0.41(0.230)^{\mathrm{a}}$ & $0.32(0.229)^{\mathrm{b}, *}$ & $0.30(0.235)^{*}$ \\
Paroxysmal pain subscore & $0.42(0.221)$ & $0.27(0.231)^{*}$ & $0.25(0.255)^{*}$ \\
Evoked pain subscore & $0.39(0.216)$ & $0.27(0.212)^{*}$ & $0.27(0.252)^{*}$ \\
Paresthesia/dysesthesia subscore & $0.42(0.233)$ & $0.30(0.210)^{*}$ & $0.30(0.239)^{*}$ \\
\hline b) Number of pain attacks & & & \\
\hline Number of pain attacks during the past 24 h, $n(\%)$ & Baseline & Week 6 & Week 12 \\
& $(n=76)$ & $(n=65)$ & $(n=59)$ \\
\hline$>20$ attacks & $6(7.9)$ & $5(7.7)$ & $4(6.8)$ \\
$11-20$ attacks & $10(13.2)$ & $10(15.4)$ & $7(11.9)$ \\
6-10 attacks & $19(25.0)$ & $12(18.5)$ & $11(18.6)$ \\
$1-5$ attacks & $28(36.8)$ & $20(30.8)$ & $14(23.7)$ \\
No attacks & $8(10.5)$ & $14(21.5)$ & $20(33.9)$ \\
Missing & $5(6.6)$ & $4(6.2)$ & $3(5.1)$ \\
\hline
\end{tabular}

NPSI Neuropathic Pain Symptom Inventory, $S D$ standard deviation

${ }^{\mathrm{a}} n=69$

${ }^{\mathrm{b}} n=60$

${ }^{*} P<0.05$ for the change from baseline

and flupirtine. Of the eight patients who reduced their dose of pregabalin, four patients tapered their dose completely, dose reductions of $75 \%$ and $50 \%$ were achieved by one patient each, and two patients did not reduce their dose. Of the three patients who reduced their dose of gabapentin, a complete dose reduction was achieved by two patients, and a dose reduction of $33.3 \%$ was achieved by the remaining patient. Responder rate 1 was $82.6 \%(19 / 23)$ at week 6 , $78.3 \%(18 / 23)$ at week 8 , and $87.0 \%(20 / 23)$ at week 12; responder rate 2 was $69.6 \%(16 / 23)$ at week $6,73.9 \%(17 / 23)$ at week 8 , and $82.6 \%$ $(19 / 23)$ at week 12. Significant decreases were observed in the mean (SD) pain intensity score from baseline (5.0 [0.21]) to week 6 (mean [SD] change from baseline, -1.3 [2.14]; $P=0.0079$ ), week $8(-1.4[2.13] ; P=0.0038)$, and week 12 $(-1.9$ [2.23]; $P=0.0005)$.

\section{Safety and Tolerability}

All patients reported at least one NTEAE. Of the 713 NTEAEs recorded, 708 were related to ongoing medical conditions or diseases present at the screening visit; the remaining five were newly occurring NTEAEs that were reported during week -1 . The most common (frequency $\geq 5 \%)$ NTEAEs reported were constipation (9.1\% [65/713]), hypertension (6.5\% [46/713]), and nausea $(6.0 \%[43 / 713])$.

A total of $68.0 \%(85 / 125)$ of patients reported at least one TEAE. A total of $67.3 \%(245 / 364)$ of the 364 TEAEs reported from week 1 to week 12 
Table 12 Percent reduction of analgesic or coanalgesic medication during the tapering substudy (substudy subset)

\begin{tabular}{ll}
\hline Percent reduction of analgesic or & Total \\
coanalgesic, $n(\%)$ & \\
\hline Any analgesic or coanalgesic $(\%)$ & $(n=23)$ \\
100.0 & $15(65.2)$ \\
75.0 & $1(4.3)$ \\
50.0 & $2(8.7)$ \\
33.3 & $2(8.7)$ \\
0 & $3(13.0)$ \\
WHO step I analgesics $(\%)$ & $(n=6)$ \\
100.0 & $6(100.0)$ \\
Coanalgesics $(\%)$ & $(n=17)$ \\
100.0 & $9(52.9)$ \\
75.0 & $1(5.9)$ \\
50.0 & $2(11.8)$ \\
33.3 & $2(11.8)$ \\
0 & $3(17.6)$ \\
Antidepressants $(\%)$ & $(n=4)$ \\
100.0 & $2(50.0)$ \\
50.0 & $1(25.0)$ \\
0 & $1(25.0)$ \\
Anticonvulsants $(\%)$ & $(n=11)$ \\
100.0 & $6(54.5)$ \\
75.0 & $1(9.1)$ \\
50.0 & $1(9.1)$ \\
33.3 & $1(9.1)$ \\
0 & $2(18.2)$ \\
\hline
\end{tabular}

WHO World Health Organization

were classified as at least possibly related to study medication. A total of $78.6 \%(286 / 364)$ of the reported TEAEs were of mild-to-moderate intensity. The TEAEs that were reported by at least 5\% of patients are summarized in Table 13. Drug withdrawal syndrome, which was likely due to physical dependence related to the previous opioid analgesic, occurred in $20.8 \%(26 / 125)$ of patients. All but one case of drug withdrawal syndrome occurred at the switch from prior WHO step III therapy between weeks 0 and 6; one patient reported additional drug withdrawal syndrome at the end of the study. A total of $50 \%$ $(13 / 26)$ of cases of drug withdrawal were reported at a single study site. The prevalence of the AEs that patients reported as the underlying reason for switching to tapentadol generally decreased from week -1 (on WHO step III opioids) to week 12 (on tapentadol PR; Fig. 7); the most common AEs reported as the underlying reason for switching to tapentadol (incidence $\geq 10 \%$ at week -1) were constipation, nausea, fatigue, and somnolence. Serious TEAEs were reported for $8.8 \%(11 / 125)$ of patients. Twelve of the 19 serious TEAEs reported were considered by the investigator to be unlikely or not related to study treatment. One additional patient experienced toxic hepatitis that was considered by the investigator to be probably related to study treatment and occurred 1 day after the end of study treatment; this TEAE was not included in the count of serious TEAEs. A total of $14.4 \%(18 / 125)$ of patients experienced TEAEs that led to study discontinuation; 15 of these 18 TEAEs were considered by the investigator to be related to study treatment. TEAEs leading to study discontinuation (incidence $\geq 1 \%$ ) included nausea $(3.2 \%$ [4/125]), vomiting $(2.4 \%$ [3/125]), drug withdrawal syndrome $(1.6 \%$ $[2 / 125])$, headache $(1.6 \%[2 / 125])$, dizziness $(1.6 \%[2 / 125])$, disturbance in attention $(1.6 \%$ [2/125]), and vertigo (1.6\% [2/125]).

Vital sign parameters, laboratory values, or physical examination findings showed no clinically relevant changes over the course of study treatment.

\section{DISCUSSION}

Results of this study were positive and indicated that tapentadol PR (50-250 mg b.i.d.) provided at least comparable effectiveness to previous WHO step III opioid analgesics for the management of 
Table 13 TEAEs reported by at least $5 \%$ of patients (safety population) $)^{\mathrm{a}, \mathrm{b}}$

\begin{tabular}{ll}
\hline $\begin{array}{l}\text { System organ class, } n(\%) \\
\text { Preferred term, } n(\%)\end{array}$ & Total \\
$(n=125)$
\end{tabular}

TEAE treatment-emergent adverse event

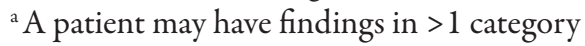

b TEAEs were coded using the Medical Dictionary for Regulatory Activities (MedDRA), version 13.1

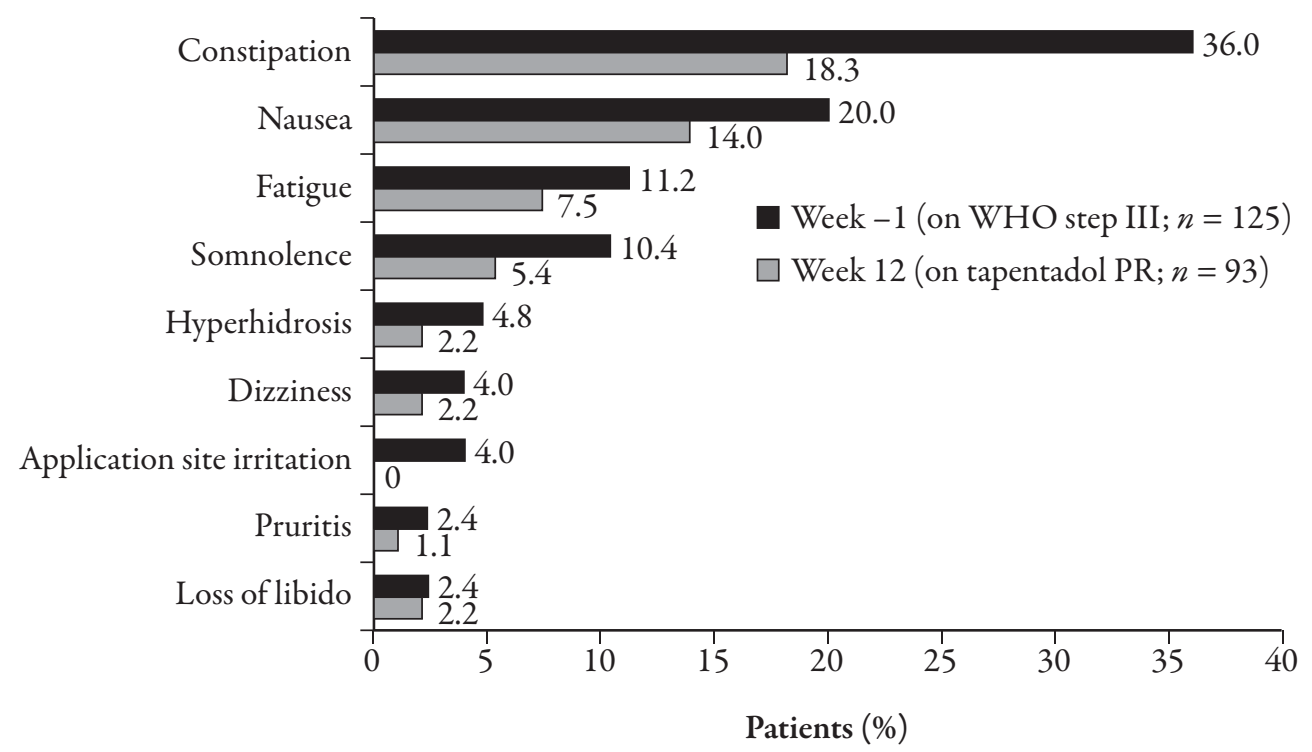

Fig. 7 Prevalence of AEs $(\geq 2 \%)$ reported as the underlying reason for switching to tapentadol at week -1 and at week 12 (safety population). ${ }^{a} A E$ adverse event, $P R$ prolonged release, $W H O$ World Health Organization. ${ }^{\text {a }}$ The prevalence of these AEs was summarized during week -1 (the week prior to titration when patients were still on WHO step III treatment) and during week 12 (the final week of tapentadol PR treatment) 
severe, chronic low back pain in patients who had responded to WHO step III opioids but showed a lack of tolerability. After 4 weeks of tapentadol PR treatment and through the remainder of the study, approximately $80 \%$ of patients had the same or less pain intensity compared with week -1 (on prior WHO step III therapy). Based on mean pain intensity scores (NRS-3), pain relief was maintained after switching from WHO step III opioids to tapentadol PR treatment; further significant improvements in mean pain intensity scores were observed with continued tapentadol PR treatment. In this study, pain relief was maintained or improved for patients who switched directly from WHO step III opioid treatment to tapentadol PR, with the majority of patients requiring no dose adjustment to reach the minimum target of titration at week 6 , and treatment discontinuations were relatively infrequent. Improvements were also observed in patient-rated measures of quality of life, health status, and function, including PGIC and CGIC ratings, the EQ-5D health status index, SF-36 scores, and the HADS anxiety and depression subscale scores. Patient satisfaction also increased substantially upon rotation to tapentadol PR treatment. At baseline, all patients rated their treatment satisfaction as "fair" or "poor," and after 6 weeks of treatment with tapentadol $\mathrm{PR}$, these ratings improved such that $72.5 \%$ of patients rated their satisfaction as "good," "very good," or "excellent." It is also noteworthy that satisfaction ratings continued to improve over the course of the study $(82.8 \%$ of patients had "good," "very good," or "excellent" ratings at week 12).

There were some differences observed in the history of low back pain between the painDETECT negative and unclear/ positive groups. On average, patients in the painDETECT unclear/positive subset consulted a doctor for their pain earlier and required more consultations, analgesic regimens, and hospitalizations for their pain than patients in the painDETECT negative subset, but patients in the painDETECT unclear/positive subset were generally off work fewer times for pain during the past year than those in the painDETECT negative subset. These results should be interpreted with caution because employment status was not documented in this study; therefore, it is possible that the differences in the reported impact of pain on time off work between the painDETECT negative and unclear/positive groups may have been related to differences in employment status (i.e., unemployed patients would not have reported that pain had an impact on time off work).

For patients who reduced or discontinued concomitant WHO step I opioids or coanalgesics in the tapering substudy, responder rates were generally in line with those observed for the overall study population. The percentage of patients with the same or less pain compared with week -1 remained relatively constant following the discontinuation of analgesics or coanalgesics in the substudy. Similar to the overall study population, significant decreases from baseline were observed in mean pain intensity scores at weeks 6,8 , and 12 for patients participating in the tapering substudy. These results indicate that these concomitant WHO step I analgesics and coanalgesics did not contribute substantially to the pain relief achieved during the study, based on the maintenance and improvement of pain relief with tapentadol alone.

For patients with low back pain with a neuropathic pain component (painDETECT unclear or positive score at baseline or screening), the effects of tapentadol treatment on the neuropathic pain component of their low back pain were evaluated in further detail. Although the painDETECT questionnaire has been recently validated for the evaluation of neuropathic 
pain over time [33], it was not validated when this study was conducted [30]. Therefore, the validated NPSI [31] was used concomitantly to measure changes in neuropathic pain symptoms. Both the painDETECT and NPSI results showed that on average, patients with a neuropathic pain component to their low back pain experienced significant improvements in symptoms of neuropathic pain. In addition, NPSI results showed that the number of pain attacks during a 24-h period decreased over the course of treatment with tapentadol PR. Results from both the current study and a previous study [22] of tapentadol PR in patients with severe, chronic low back pain with a neuropathic component indicate that painDETECT classification can be altered with effective treatment. In the previous study in patients with severe, chronic low back pain, all patients with a neuropathic component to their low back pain (a painDETECT unclear or positive score) reached a painDETECT negative score over the course of treatment with tapentadol PR [22]. That study involved patients who had not responded to their previous therapy of WHO step I or II opioids or no regular analgesic treatment [22]. In the current study, patients with a painDETECT positive score $(>18)$ at baseline achieved an unclear score $(<18)$ and patients with a painDETECT unclear score at baseline achieved a negative score $(<12)$ over the course of treatment with tapentadol PR. This study used a selected population of patients who had responded to WHO step III opioid therapy (as evidenced by NRS-3 scores); patients in this study with painDETECT unclear or positive scores had achieved pain relief with their prior therapy, but still suffered from neuropathic pain symptoms that WHO step III opioids did not control. Without a washout period, estimations of the neuropathic pain component using the painDETECT questionnaire and NPSI may have been affected by effective prior therapy, which may have decreased neuropathic pain symptoms. Nevertheless, significant improvements were observed in neuropathic pain symptoms (based on the NPSI and painDETECT) with tapentadol treatment in the current study; these improvements are particularly striking because the patients in this study had already achieved adequate pain relief with their prior analgesic regimen. In this study, the NPSI referred to pain radiating towards or into the leg, which is characteristic of sciatica (one of the primary presenting symptoms of lumbosacral radiculopathy) [34]. Double-blind studies of standard regimens used for neuropathic pain (e.g., pregabalin, tricyclic antidepressants, opioids) have shown somewhat limited efficacy in chronic painful radiculopathy [10]. The improvements in neuropathic pain symptoms for pain radiating towards or into the leg in the current study suggest that tapentadol may be useful for the management of pain typically associated with radiculopathy [35]. These results may reflect the benefit of a combination of opioid action and noradrenaline reuptake inhibition provided by tapentadol [36].

The tapering of WHO step I analgesics and coanalgesics in the substudy from week 9 to 12 could have led to artificially induced pain peaks that would have affected effectiveness, quality of life, and function results. For all effectiveness, quality of life, and function measures, outcomes were consistent when results from weeks 9 through 12 for patients who participated in the tapering substudy were included and when these results were excluded, indicating that the tapering of WHO step I analgesics or coanalgesics in the substudy had no clinically relevant impact on the evaluated outcomes.

For patients showing a poor response to an opioid analgesic or who experience intolerable side effects, opioid switching is often used to improve pain relief or tolerability [37, 38]. 
Determining the appropriate ratio, when switching from one opioid analgesic to another, is critical for successful conversion, particularly for patients who showed a poor response to the prior opioid or for those who are switching from high doses of opioids [37]. Drug overdose may result in intolerable side effects with the new opioid analgesic, while underdosing may result in the occurrence of withdrawal symptoms. In a study of patients with chronic, nonmalignant pain, the rate of withdrawal was $32 \%$ for patients switching from one PR opioid to another and $44 \%$ for patients switching from an IR opioid to a PR opioid [39]. There is a lack of strong evidence related to opioid switching, particularly with respect to adequate equipotency data $[37,38]$.

The current multicenter, multinational, open-label phase $3 \mathrm{~b}$ study was designed to provide evidence for the efficacy and tolerability of tapentadol, when switching directly from a strong opioid analgesic that provided adequate analgesia but poor tolerability. Based on the prevalence of AEs reported as the underlying reason for switching to tapentadol, improvements in tolerability were observed with tapentadol PR relative to previous WHO step III analgesic regimens. In the current study, 21\% of patients reported a TEAE of drug withdrawal. Although many of these cases may be attributed to a site-related effect (because $50 \%$ of cases were observed at a single study site), this outcome may need to be taken into consideration when switching patients from strong opioid therapy to tapentadol PR. In this study, starting doses of tapentadol (which were based on the MEDs of the previous WHO step III opioids) were approximately $30-75 \%$ lower than the calculated dose of the previous opioid; given that the doses of tapentadol were at least 30\% lower than the MEDs of previous opioids, the lower $\mu$-opioid component of tapentadol compared with prior
WHO step III opioids may also need to be taken into account for equianalgesic conversions in patients at risk of developing withdrawal. In the higher MED dose ranges, equianalgesic ratios decreased in favor of tapentadol, suggesting that a low cross-tolerance to classical opioids allowed patients to overcome the dose excesses caused by tolerance to the previous opioid by rotation to tapentadol. This finding is also in line with a proposed lower potential for the development of tolerance with tapentadol treatment.

There were several limitations to this study. The sample size was lower than initially planned because of the early termination of the study due to slow recruitment; a higher sample size might have contributed to a more accurate calculation of equianalgesic ratios. This was an effectivenessand practice-oriented open-label study without a placebo or active comparator. As described previously, screening and baseline painDETECT and NPSI results may have been affected by prior treatment. Despite any potential limitations, it is recognized more and more by the scientific community that real-world effectiveness trials are of great value, as they are highly related to routine clinical practice and avoid the artificial settings and patient selection requirements that are commonly required for trials driven by regulatory guidelines.

\section{CONCLUSIONS}

Results of this open-label, phase $3 \mathrm{~b}$ study support those of previous, randomized, doubleblind, controlled, phase 3 studies of tapentadol PR for moderate-to-severe, chronic low back pain [18] and neuropathic pain (painful diabetic peripheral neuropathy) [20]. Overall, results indicate that direct rotation from previous $\mathrm{WHO}$ step III opioid therapy to tapentadol PR could be achieved without compromising pain relief in a strong opioid responder population, allowing 
for even further reduction of pain intensity. Equianalgesic ratios could be calculated in the current study for tapentadol to oxycodone, buprenorphine, fentanyl, morphine, and hydromorphone; the equianalgesic ratios calculated for tapentadol to oxycodone were in line with those observed in previous randomized, double-blind, placebo- and activecontrolled, phase 3 studies of tapentadol PR for chronic pain, in which oxycodone was the main comparator [17, 18]. Furthermore, tapentadol PR was associated with improvements in health status, quality of life, treatment satisfaction, function, neuropathic pain symptoms, and tolerability relative to prior WHO step III opioid analgesics.

\section{ACKNOWLEDGMENTS}

This study and page charges were sponsored by Medical Affairs, Grünenthal Europe \& Australia, Grünenthal GmbH, Aachen, Germany. Editorial support for the writing of this manuscript was provided by Megan Knagge, PhD, of MedErgy, and was funded by Medical Affairs, Grünenthal GmbH, Aachen, Germany. The authors retained full editorial control over the content of the manuscript.

Ilona Steigerwald is the guarantor for this article, and takes responsibility for the integrity of the work as a whole.

Conflict of interest. Michael Schäfer has served as a consultant for Grunenthal. Dietmar Falke is an employee of Grünenthal $\mathrm{GmbH}$. Ilona Steigerwald is an employee of Grünenthal GmbH. Rafael Gálvez declares he has no conflict of interest. Guy Hans declares he has no conflict of interest.

Open Access. This article is distributed under the terms of the Creative Commons Attribution Noncommercial License which permits any noncommercial use, distribution, and reproduction in any medium, provided the original author(s) and source are credited.

\section{APPENDIX}

Table A1 Responder rates 1 and 2 at baseline and at weeks 6 and 12 using observed-case analysis (with and without the week 9-12 data for the tapering substudy population) and using the LOCF (without the week 9-12 data for the tapering substudy population) - main analysis population

\begin{tabular}{llllll}
\hline & \multicolumn{2}{l}{ Observed-case analysis } & \multicolumn{2}{l}{ LOCF } & \\
\cline { 2 - 5 } & Week 6 & Week 12 & & Week 6 & Week 12 \\
\hline Responder rate, $n$ (\%) & & $\begin{array}{l}\text { With } \\
\text { substudy }\end{array}$ & $\begin{array}{l}\text { Without } \\
\text { substudy }\end{array}$ & & $\begin{array}{l}\text { Without } \\
\text { substudy }\end{array}$ \\
\hline painDETECT negative & $(n=37)$ & $(n=34)$ & $(n=28)$ & $(n=45)$ & $(n=39)$ \\
Responder rate 1 & $30(81.1)$ & $31(91.2)$ & $25(89.3)$ & $35(77.8)$ & $32(82.1)$ \\
Responder rate 2 & $23(62.2)$ & $25(73.5)$ & $20(71.4)$ & $24(53.3)$ & $21(53.8)$ \\
painDETECT unclear/positive & $(n=65)$ & $(n=59)$ & $(n=42)$ & $(n=76)$ & $(n=59)$ \\
Responder rate 1 & $52(80.0)$ & $45(76.3)$ & $31(73.8)$ & $59(77.6)$ & $41(69.5)$ \\
Responder rate 2 & $42(64.6)$ & $44(74.6)$ & $30(71.4)$ & $46(60.5)$ & $35(59.3)$ \\
Total & $(n=102)$ & $(n=93)$ & $(n=70)$ & $(n=121)$ & $(n=98)$ \\
Responder rate 1 & $82(80.4)$ & $76(81.7)$ & $56(80.0)$ & $94(77.7)$ & $73(74.5)$ \\
Responder rate 2 & $65(63.7)$ & $69(74.2)$ & $50(71.4)$ & $70(57.9)$ & $56(57.1)$ \\
\hline
\end{tabular}

LOCF last observation carried forward 
Table A2 Mean (SD) pain intensity scores (11-point NRS-3) at baseline and at weeks 6 and 12 using observed-case analysis (with and without the week 9-12 data for the tapering substudy population) and using the LOCF (without the week 9-12 data for the tapering substudy population) - main analysis population

\begin{tabular}{lllllll}
\hline & & \multicolumn{2}{l}{ Observed-case analysis } & & LOCF \\
\cline { 3 - 7 } & Baseline & Week 6 & Week 12 & & Week 6 & Week 12 \\
\hline Pain intensity & & $\begin{array}{l}\text { With } \\
\text { substudy } \\
\end{array}$ & $\begin{array}{l}(n=122) \\
\text { Mean (SD) pain intensity }\end{array}$ & $\begin{array}{l}(n=101) \\
\text { Substudy } \\
(n=70)\end{array}$ & $\begin{array}{l}\text { Without } \\
\text { substudy } \\
(n=98)\end{array}$ \\
\hline
\end{tabular}

LOCF last observation carried forward, NRS-3 numerical rating scale-3, $S D$ standard deviation. ${ }^{*} P<0.05$ for the change from baseline

Table A3 Subject satisfaction with treatment ratings at baseline and at weeks 6 and 12 using observed-case analysis (with and without the week 9-12 data for the tapering substudy population) and using the LOCF (without the week 9-12 data for the tapering substudy population) - main analysis population

\begin{tabular}{|c|c|c|c|c|c|c|}
\hline \multirow[b]{3}{*}{ Satisfaction rating, $n(\%)$} & \multirow{3}{*}{$\begin{array}{l}\text { Baseline } \\
(n=123)\end{array}$} & \multicolumn{3}{|c|}{ Observed-case analysis } & \multicolumn{2}{|l|}{ LOCF } \\
\hline & & Week 6 & Week 12 & & Week 6 & Week 12 \\
\hline & & $(n=102)$ & $\begin{array}{l}\text { With } \\
\text { substudy } \\
(n=93)\end{array}$ & $\begin{array}{l}\text { Without } \\
\text { substudy } \\
(n=70)\end{array}$ & $(n=121)$ & $\begin{array}{l}\text { Without } \\
\text { substudy } \\
(n=98)\end{array}$ \\
\hline Excellent & 0 & $2(2.0)$ & $2(2.2)$ & $2(2.9)$ & $2(1.7)$ & $2(2.0)$ \\
\hline Very good & 0 & $25(24.5)$ & $32(34.4)$ & $22(31.4)$ & $25(20.7)$ & $22(22.4)$ \\
\hline Good & 0 & $47(46.1)$ & $43(46.2)$ & $34(48.6)$ & $51(42.1)$ & $40(40.8)$ \\
\hline Fair & $92(74.8)$ & $21(20.6)$ & $11(11.8)$ & $9(12.9)$ & $30(24.8)$ & $23(23.5)$ \\
\hline Poor & $30(24.4)$ & $5(4.9)$ & $4(4.3)$ & $2(2.9)$ & $13(10.7)$ & $11(11.2)$ \\
\hline Missing & $1(0.8)$ & $2(2.0)$ & $1(1.1)$ & $1(1.4)$ & 0 & 0 \\
\hline
\end{tabular}

LOCF last observation carried forward

Table A4 PGIC and CGIC ratings at baseline and at weeks 6 and 12 using observed-case analysis (with and without the week 9-12 data for the tapering substudy population) and using the LOCF (without the week 9-12 data for the tapering substudy population) - main analysis population

\begin{tabular}{|c|c|c|c|c|c|}
\hline \multirow[b]{3}{*}{ Rating, $n(\%)$} & \multicolumn{3}{|c|}{ Observed-case analysis } & \multicolumn{2}{|l|}{ LOCF } \\
\hline & Week 6 & Week 12 & & Week 6 & Week 12 \\
\hline & $(n=102)$ & $\begin{array}{l}\text { With } \\
\text { substudy } \\
(n=93)\end{array}$ & $\begin{array}{l}\text { Without } \\
\text { substudy } \\
(n=70)\end{array}$ & $(n=120)$ & $\begin{array}{l}\text { Without } \\
\text { substudy } \\
(n=97)\end{array}$ \\
\hline \multicolumn{6}{|l|}{ PGIC } \\
\hline Very much improved & $5(4.9)$ & $9(9.7)$ & $5(7.1)$ & $5(4.2)$ & $5(5.2)$ \\
\hline Much improved & $29(28.4)$ & $34(36.6)$ & $24(34.3)$ & $30(25.0)$ & $25(25.8)$ \\
\hline Minimally improved & $47(46.1)$ & $38(40.9)$ & $31(44.3)$ & $48(40.0)$ & $35(36.1)$ \\
\hline No change & $11(10.8)$ & $9(9.7)$ & $8(11.4)$ & $17(14.2)$ & $14(14.4)$ \\
\hline Minimally worse & $6(5.9)$ & $2(2.2)$ & $1(1.4)^{\prime}$ & $9(7.5)$ & $8(8.2)$ \\
\hline Much worse & $3(2.9)$ & $1(1.1)$ & $1(1.4)$ & $10(8.3)$ & $9(9.3)$ \\
\hline Very much worse & 0 & 0 & 0 & $1(0.8)$ & $1(1.0)$ \\
\hline Missing & $1(1.0)$ & 0 & 0 & 0 & 0 \\
\hline \multicolumn{6}{|l|}{ CGIC } \\
\hline Very much improved & $5(4.9)$ & $11(11.8)$ & $5(7.1)$ & $5(4.2)$ & $5(5.2)$ \\
\hline Much improved & $39(38.2)$ & $41(44.1)$ & $31(44.3)$ & $40(33.3)$ & $32(33.0)$ \\
\hline Minimally improved & $38(37.3)$ & $29(31.2)$ & $24(34.3)$ & $40(33.3)$ & $30(30.9)$ \\
\hline No change & $12(11.8)$ & $8(8.6)$ & $8(11.4)$ & $16(13.3)$ & $14(14.4)$ \\
\hline Minimally worse & $5(4.9)$ & $3(3.2)$ & $1(1.4)$ & $12(10.0)$ & $9(9.3)$ \\
\hline Much worse & $2(2.0)$ & $1(1.1)$ & $1(1.4)$ & $7(5.8)$ & $7(7.2)$ \\
\hline Very much worse & 0 & 0 & 0 & 0 & 0 \\
\hline Missing & $1(1.0)$ & 0 & 0 & 0 & 0 \\
\hline
\end{tabular}

CGIC clinician global impression of change, $L O C F$ last observation carried forward, $P G I C$ patient global impression of change 
Table A5 Mean (SD) EQ-5D Health Status Index Score at baseline and at weeks 6 and 12 using observed-case analysis (with and without the week 9-12 data for the tapering substudy population) and using the LOCF (without the week 9-12 data for the tapering substudy population) - main analysis population

\begin{tabular}{lllllll}
\hline & & \multicolumn{2}{l}{ Observed-case analysis } & \multicolumn{3}{c}{ LOCF } \\
\cline { 3 - 7 } & Baseline & Week 6 & Week 12 & Week 6 & Week 12 \\
\hline & & & With & Without \\
substudy & $\begin{array}{l}\text { substudy } \\
(n=70)\end{array}$ & $(n=118)$ & $\begin{array}{l}\text { Without } \\
\text { substudy } \\
(n=95)\end{array}$ \\
\hline EQ-5D health status index score & $0.39(0.307)$ & $0.55(0.246)^{*}$ & $0.57(0.252)^{*}$ & $0.57(0.242)^{*}$ & $0.53(0.251)^{*}$ & $0.52(0.255)^{*}$ \\
\hline
\end{tabular}

EQ-5D EuroQol-5 Dimension, $L O C F$ last observation carried forward, $S D$ standard deviation

${ }^{*} P<0.0001$ for the change from baseline

Table A6 Mean (SD) SF-36 scores at baseline and at weeks 6 and 12 using observed-case analysis (with and without the week 9-12 data for the tapering substudy population) and using the LOCF (without the week 9-12 data for the tapering substudy population) - main analysis population

\begin{tabular}{|c|c|c|c|c|c|c|}
\hline \multirow[b]{3}{*}{ Subscale or summary } & \multirow[b]{2}{*}{ Baseline } & \multicolumn{3}{|c|}{ Observed-case analysis } & \multicolumn{2}{|l|}{ LOCF } \\
\hline & & Week 6 & Week 12 & & Week 6 & Week 12 \\
\hline & $(n=120)$ & $(n=99)$ & $\begin{array}{l}\text { With } \\
\text { substudy } \\
(n=93)\end{array}$ & $\begin{array}{l}\text { Without } \\
\text { substudy } \\
(n=70)\end{array}$ & $(n=118)$ & $\begin{array}{l}\text { Without } \\
\text { substudy } \\
(n=95)\end{array}$ \\
\hline Role - physical $^{a}$ & $13.4(25.17)$ & $21.7(29.81)^{*}$ & $24.2(35.25)^{*}$ & $23.9(35.73)^{*}$ & $18.2(28.43)$ & $18.4(32.25)$ \\
\hline Physical functioning & $33.5(19.94)$ & $42.4(21.52)^{*}$ & $43.8(24.47)^{*}$ & $43.4(23.18)^{*}$ & $40.3(21.74)^{*}$ & $40.6(23.08)^{*}$ \\
\hline Bodily pain ${ }^{b}$ & $28.8(14.30)$ & $40.3(16.98)^{*}$ & $42.9(19.00)^{*}$ & $39.8(17.83)^{*}$ & $37.8(17.34)^{*}$ & $36.5(17.51)^{*}$ \\
\hline General health ${ }^{c}$ & $41.3(20.32)$ & $48.7(20.92)^{*}$ & $48.1(22.01)^{*}$ & $48.3(22.70)^{*}$ & $46.3(21.15)^{*}$ & $44.5(22.07)^{*}$ \\
\hline Vitality & $32.3(17.75)$ & $42.2(19.64)^{*}$ & $45.1(22.66)^{*}$ & $44.0(22.03)^{*}$ & $40.0(19.90)^{*}$ & $40.1(21.49)^{*}$ \\
\hline Social functioning ${ }^{\mathrm{b}}$ & $44.0(28.30)$ & $52.7(26.08)^{*}$ & $55.6(27.68)^{*}$ & $55.0(25.22)^{*}$ & $48.4(27.23)$ & $48.9(26.65)$ \\
\hline Role - emotional ${ }^{a}$ & $42.6(44.24)$ & $41.4(40.44)$ & $56.2(42.21)^{*}$ & $55.6(41.10)^{*}$ & $43.2(41.42)$ & $51.6(42.05)$ \\
\hline Mental health & $54.3(19.32)$ & $60.2(20.52)^{*}$ & $65.3(22.76)^{*}$ & $65.3(22.26)^{*}$ & $58.0(20.69)^{*}$ & $60.7(22.15)^{*}$ \\
\hline Physical component ${ }^{\mathrm{b}}$ & $27.9(7.07)$ & $32.6(7.93)^{*}$ & $31.7(8.83)^{*}$ & $31.3(8.95)^{*}$ & $31.4(8.13)^{*}$ & $30.0(8.54)^{*}$ \\
\hline Mental component ${ }^{\mathrm{b}}$ & $41.2(11.97)$ & $43.1(11.47)$ & $46.7(12.55)^{*}$ & $46.6(11.99)^{*}$ & $42.3(11.46)$ & $44.4(11.83)^{*}$ \\
\hline
\end{tabular}

$B L$ baseline, $L O C F$ last observation carried forward, $S D$ standard deviation, $S F-36$ Short Form-36, $W$ week

${ }^{a} \mathrm{BL}, n=119$; observed-case analysis: W6, $n=99$; W12 (with substudy), $n=92$; W12 (without substudy), $n=69$; LOCF:

W6, $n=118$; W 12 (without substudy), $n=95$

${ }^{\mathrm{b}} \mathrm{BL}, n=120$; observed-case analysis: W6, $n=101$; W 12 (with substudy), $n=93$; W 12 (without substudy), $n=70$; LOCF:

W6, $n=118 ;$ W 12 (without substudy), $n=95$

' BL, $n=120$; observed-case analysis: W6, $n=100$; W12 (with substudy), $n=93$; W12 (without substudy), $n=70$; LOCF:

$\mathrm{W} 6, n=118$; W 12 (without substudy), $n=95$

${ }^{*} P<0.05$ for the change from baseline 
Table A7 Mean (SD) HADS anxiety and depression subscale scores at baseline and at weeks 6 and 12 using observed-case analysis (with and without the week 9-12 data for the tapering substudy population) and using the LOCF (without the week 9-12 data for the tapering substudy population) - main analysis population

\begin{tabular}{|c|c|c|c|c|c|c|}
\hline & \multirow[b]{2}{*}{ Baseline } & \multicolumn{3}{|c|}{ Observed-case analysis } & \multicolumn{2}{|l|}{ LOCF } \\
\hline & & Week 6 & Week 12 & & Week 6 & Week 12 \\
\hline Subscale & & & $\begin{array}{l}\text { With } \\
\text { substudy }\end{array}$ & $\begin{array}{l}\text { Without } \\
\text { substudy }\end{array}$ & & $\begin{array}{l}\text { Without } \\
\text { substudy }\end{array}$ \\
\hline painDETECT negative & $(n=45)$ & $(n=35)$ & $(n=34)$ & $(n=28)$ & $(n=44)$ & $(n=38)$ \\
\hline Anxiety subscale & $5.9(4.21)$ & $5.1(3.69)$ & $3.8(3.26)^{*}$ & $3.8(3.30)^{*}$ & $5.4(3.92)$ & $4.5(3.78)^{*}$ \\
\hline Depression subscale & $6.2(4.40)$ & $4.8(3.46)$ & $4.6(3.42)^{*}$ & $4.6(3.33)$ & $5.7(4.04)$ & $5.7(4.04)$ \\
\hline painDETECT unclear/positive & $(n=73)$ & $(n=64)$ & $(n=59)$ & $(n=42)$ & $(n=73)$ & $(n=56)$ \\
\hline Anxiety subscale & $8.5(3.96)$ & $7.3(4.02)^{*}$ & $7.1(4.01)^{*}$ & $6.9(4.12)^{*}$ & $7.5(4.08)^{*}$ & $7.3(4.13)^{*}$ \\
\hline Depression subscale & $8.9(4.33)$ & $8.0(4.14)^{*}$ & $8.1(4.67)$ & $7.9(4.36)$ & $8.4(4.30)^{*}$ & $8.5(4.39)$ \\
\hline Total & $(n=118)$ & $(n=99)$ & $(n=93)$ & $(n=70)$ & $(n=117)$ & $(n=94)$ \\
\hline Anxiety subscale & $7.5(4.23)$ & $6.5(4.03)^{*}$ & $5.9(4.06)^{*}$ & $5.7(4.09)^{*}$ & $6.7(4.13)^{*}$ & $6.1(4.20)^{*}$ \\
\hline Depression subscale & $7.9(4.54)$ & $6.8(4.17)^{*}$ & $6.8(4.57)^{*}$ & $6.6(4.28)^{*}$ & $7.4(4.38)^{*}$ & $7.3(4.45)$ \\
\hline
\end{tabular}

HADS Hospital Anxiety and Depression Scale, $L O C F$ last observation carried forward, $S D$ standard deviation

${ }^{*} P<0.05$ for the change from baseline

Table A8 NPSI results in the baseline painDETECT unclear/positive subset: (a) mean (SD) NPSI overall feeling and subscores, (b) total number of pain attacks within the past $24 \mathrm{~h}$ (with and without the tapering substudy population) and using the LOCF (without the tapering substudy population) - main analysis population

a) Overall feeling and subscores

\begin{tabular}{|c|c|c|c|c|c|c|}
\hline & & Observed-cas & analysis & & LOCF & \\
\hline & Baseline & Week 6 & Week 12 & & Week 6 & Week 12 \\
\hline Mean (SD) NPSI score & $(n=71)$ & $(n=61)$ & $\begin{array}{l}\text { With } \\
\text { substudy } \\
(n=56)\end{array}$ & $\begin{array}{l}\text { Without } \\
\text { substudy } \\
(n=39)\end{array}$ & $(n=72)$ & $\begin{array}{l}\text { Without } \\
\text { substudy } \\
(n=55)\end{array}$ \\
\hline Overall feeling score ${ }^{\mathrm{a}}$ & $0.41(0.158)$ & $0.30(0.178)^{*}$ & $0.28(0.211)^{*}$ & $0.32(0.217)^{*}$ & $0.30(0.177)^{*}$ & $0.32(0.196)^{*}$ \\
\hline Burning pain subscore & $0.41(0.284)$ & $0.32(0.273)^{*}$ & $0.27(0.281)^{*}$ & $0.30(0.291)^{*}$ & $0.33(0.259)^{*}$ & $0.32(0.267)^{*}$ \\
\hline Pressing pain subscore ${ }^{a}$ & $0.41(0.230)$ & $0.32(0.229)^{*}$ & $0.30(0.235)^{*}$ & $0.34(0.239)$ & $0.31(0.228)^{*}$ & $0.33(0.232)^{*}$ \\
\hline Paroxysmal pain subscore & $0.42(0.221)$ & $0.27(0.231)^{*}$ & $0.25(0.255)^{*}$ & $0.30(0.264)^{*}$ & $0.28(0.240)^{*}$ & $0.30(0.258)^{*}$ \\
\hline Evoked pain subscore & $0.39(0.216)$ & $0.27(0.212)^{*}$ & $0.27(0.252)^{*}$ & $0.33(0.268)$ & $0.28(0.206)^{*}$ & $0.32(0.243)$ \\
\hline $\begin{array}{l}\text { Paresthesia/dysesthesia } \\
\text { subscore }\end{array}$ & $0.42(0.233)$ & $0.30(0.210)^{*}$ & $0.30(0.239)^{*}$ & $0.32(0.237)^{*}$ & $0.33(0.225)^{*}$ & $0.34(0.252)^{*}$ \\
\hline
\end{tabular}

b) Number of pain attacks

\begin{tabular}{|c|c|c|c|c|c|c|}
\hline & \multirow[b]{2}{*}{ Baseline } & \multicolumn{3}{|c|}{ Observed-case analysis } & \multicolumn{2}{|l|}{ LOCF } \\
\hline & & Week 6 & Week 12 & & Week 6 & Week 12 \\
\hline $\begin{array}{l}\text { Number of pain attacks } \\
\text { during the past } 24 \mathrm{~h}, n(\%)\end{array}$ & $(n=76)$ & $(n=65)$ & $\begin{array}{l}\text { With } \\
\text { substudy } \\
(n=59)\end{array}$ & $\begin{array}{l}\text { Without } \\
\text { substudy } \\
(n=42)\end{array}$ & $(n=74)$ & $\begin{array}{l}\text { Without } \\
\text { substudy } \\
(n=57)\end{array}$ \\
\hline$>20$ attacks & $6(7.9)$ & $5(7.7)$ & $4(6.8)$ & $3(7.1)$ & $7(9.5)$ & $5(8.8)$ \\
\hline $11-20$ attacks & $10(13.2)$ & $10(15.4)$ & $7(11.9)$ & $4(9.5)$ & $12(16.2)$ & $8(14.0)$ \\
\hline $6-10$ attacks & $19(25.0)$ & $12(18.5)$ & $11(18.6)$ & $9(21.4)$ & $14(18.9)$ & $11(19.3)$ \\
\hline $1-5$ attacks & $28(36.8)$ & $20(30.8)$ & $14(23.7)$ & $12(28.6)$ & $23(31.1)$ & $15(26.3)$ \\
\hline No attacks & $8(10.5)$ & $14(21.5)$ & $20(33.9)$ & $11(26.2)$ & $16(21.6)$ & $16(28.1)$ \\
\hline Missing & $5(6.6)$ & $4(6.2)$ & $3(5.1)$ & $3(7.1)$ & $2(2.7)$ & $2(3.5)$ \\
\hline
\end{tabular}

BL baseline, LOCF last observation carried forward, NPSI Neuropathic Pain Symptom Inventory, SD standard deviation, $W$ week

${ }^{a} \mathrm{BL}, n=69$; observed-case analysis: W6, $n=60$; W12 (with substudy), $n=56$; W 12 (without substudy), $n=39$; LOCF:

W6, $n=72$; W12 (without substudy), $n=55$

${ }^{*} P<0.05$ for the change from baseline 


\section{REFERENCES}

1. Chou R, Qaseem A, Snow V, et al. Diagnosis and treatment of low back pain: a joint clinical practice guideline from the American College of Physicians and the American Pain Society. Ann Intern Med. 2007;147:478-91.

2. Chou R, Huffman LH. Medications for acute and chronic low back pain: a review of the evidence for an American Pain Society/American College of Physicians clinical practice guideline. Ann Intern Med. 2007;147:505-14.

3. Christo PJ. Opioid effectiveness and side effects in chronic pain. Anesthesiol Clin North America. 2003;21:699-713.

4. Moore RA, McQuay HJ. Prevalence of opioid adverse events in chronic non-malignant pain: systematic review of randomised trials of oral opioids. Arthritis Res Ther. 2005;7:R1046-51.

5. Ballantyne JC. Opioids for chronic nonterminal pain. South Med J. 2006;99:1245-55.

6. Gregorian RS, Jr., Gasik A, Kwong WJ, Voeller S, Kavanagh S. Importance of side effects in opioid treatment: a trade-off analysis with patients and physicians. J Pain. 2010;11:1095-108.

7. Porreca F, Ossipov MH. Nausea and vomiting side effects with opioid analgesics during treatment of chronic pain: mechanisms, implications, and management options. Pain Med. 2009;10:654-62.

8. Bell TJ, Panchal SJ, Miaskowski C, Bolge SC, Milanova T, Williamson R. The prevalence, severity, and impact of opioid-induced bowel dysfunction: results of a US and European Patient Survey (PROBE 1). Pain Med. 2009;10:35-42.

9. Duensing L, Eksterowicz N, Macario A, Brown M, Stern L, Ogbonnaya A. Patient and physician perceptions of treatment of moderate-to-severe chronic pain with oral opioids. Curr Med Res Opin. 2010;26:1579-85.

10. Attal N, Cruccu G, Baron R, et al. EFNS guidelines on the pharmacological treatment of neuropathic pain: 2010 revision. Eur J Neurol. 2010;17:1113-1e88.

11. Bartleson JD. Evidence for and against the use of opioid analgesics for chronic nonmalignant low back pain: a review. Pain Med. 2002;3:260-71.

12. Slatkin NE. Opioid switching and rotation in primary care: implementation and clinical utility. Curr Med Res Opin. 2009;25:2133-50.
13. Varrassi G, Muller-Schwefe GH. The international CHANGE PAIN physician survey: does specialism influence the perception of pain and its treatment? Curr Med Res Opin. 2012;28:823-31.

14. Chaparro LE, Wiffen PJ, Moore RA, Gilron I. Combination pharmacotherapy for the treatment of neuropathic pain in adults. Cochrane Database Syst Rev. 2012;7:CD008943.

15. Tzschentke TM, Christoph T, Kögel B, et al. (-)-(1R,2R)-3-(3-Dimethylamino-1-ethyl-2-methylpropyl)-phenol hydrochloride (tapentadol $\mathrm{HCl}$ ): a novel m-opioid receptor agonist/norepinephrine reuptake inhibitor with broad-spectrum analgesic properties. J Pharmacol Exp Ther. 2007;323:265-76.

16. Tzschentke TM, De Vry J, Terlinden R, et al. Tapentadol hydrochloride. Analgesic, mu-opioid receptor agonist, noradrenaline reuptake inhibitor. Drugs Future. 2006;31:1053-61.

17. Afilalo M, Etropolski MS, Kuperwasser B, et al. Efficacy and safety of tapentadol extended release compared with oxycodone controlled release for the management of moderate to severe chronic pain related to osteoarthritis of the knee: a randomized, double-blind, placebo- and active-controlled phase III study. Clin Drug Invest. 2010;30:489-505.

18. Buynak R, Shapiro DY, Okamoto A, et al. Efficacy and safety of tapentadol extended release for the management of chronic low back pain: results of a prospective, randomized, double-blind, placeboand active-controlled phase III study. Expert Opin Pharmacother. 2010;11:1787-804.

19. Lange B, Kuperwasser B, Okamoto A, et al. Efficacy and safety of tapentadol prolonged release for chronic osteoarthritis pain and low back pain. Adv Ther. 2010;27:381-99.

20. Schwartz S, Etropolski M, Shapiro DY, et al. Safety and efficacy of tapentadol ER in patients with painful diabetic peripheral neuropathy: results of a randomized-withdrawal, placebo-controlled trial. Curr Med Res Opin. 2011;27:151-62.

21. Wild JE, Grond S, Kuperwasser B, et al. Longterm safety and tolerability of tapentadol extended release for the management of chronic low back pain or osteoarthritis pain. Pain Pract. 2010;10:416-27.

22. Steigerwald I, Müller M, Davies A, et al. Effectiveness and safety of tapentadol prolonged release for severe, chronic low back pain with or without a neuropathic pain component: results of an open-label, phase 3b study. Curr Med Res Opin. 2012;28:911-36. 
23. Dworkin RH, Turk DC, Farrar JT, et al. Core outcome measures for chronic pain clinical trials: IMMPACT recommendations. Pain. 2005;113:9-19.

24. Farrar JT, Young JP, Jr., LaMoreaux L, Werth JL, Poole RM. Clinical importance of changes in chronic pain intensity measured on an 11-point numerical pain rating scale. Pain. 2001;94:149-58.

25. Schneider LS, Olin JT, Doody RS, et al. Validity and reliability of the Alzheimer's Disease Cooperative Study-Clinical Global Impression of Change. The Alzheimer's Disease Cooperative Study. Alzheimer Dis Assoc Disord. 1997;11:S22-S32.

26. Selai CE, Trimble MR, Price MJ, Remak E. Evaluation of health status in epilepsy using the EQ-5D questionnaire: a prospective, observational, 6-month study of adjunctive therapy with anti-epileptic drugs. Curr Med Res Opin. 2005;21:733-9.

27. Ware JE, Jr., Donald Sherbourne C. The MOS 36-item Short-Form Health Survey (SF-36). I. Conceptual framework and item selection. Med Care. 1992;30:473-83.

28. Bjelland I, Dahl AA, Haug TT, Neckelmann D. The validity of the Hospital Anxiety and Depression Scale. An updated literature review. J Psychosom Res. 2002;52:69-77.

29. Snaith RP. The Hospital Anxiety and Depression Scale. Health Qual Life Outcomes. 2003;1:29.

30. Freynhagen R, Baron R, Gockel U, Tolle TR. painDETECT: a new screening questionnaire to identify neuropathic components in patients with back pain. Curr Med Res Opin. 2006;22:1911-20.
31. Bouhassira D, Attal N, Fermanian J, et al. Development and validation of the Neuropathic Pain Symptom Inventory. Pain. 2004;108:248-57.

32. Freynhagen R, Baron R. The evaluation of neuropathic components in low back pain. Curr Pain Headache Rep. 2009;13:185-90.

33. Tolle TR, Gockel U, Baron R, et al. Test and re-test of the painDETECT questionnaire (PD-Q). Abstract accepted for presentation at: the 4th International Congress on Neuropathic Pain (NeuPSIG 2013); 23-26 May, 2013; Toronto, Canada.

34. Tarulli AW, Raynor EM. Lumbosacral radiculopathy. Neurol Clin. 2007;25:387-405.

35. Biondi DM, Xiang J, Benson C, Etropolski M, Moskovitz B, Rauschkolb C. Tapentadol IR versus oxycodone IR for treatment of acute low back pain. Pain Physician. 2012. In press.

36. Schröder W, Tzschentke TM, Terlinden R, et al. Synergistic interaction between the two mechanisms of action of tapentadol in analgesia. J Pharmacol Exp Ther. 2011;337:312-20.

37. Mercadante S, Bruera E. Opioid switching: a systematic and critical review. Cancer Treat Rev. 2006;32:304-15.

38. Quigley C. Opioid switching to improve pain relief and drug tolerability. Cochrane Database Syst Rev. 2004;CD004847.

39. Thomsen AB, Becker N, Eriksen J. Opioid rotation in chronic non-malignant pain patients. A retrospective study. Acta Anaesthesiol Scand. 1999;43:918-23. 